\title{
Random Orderings and Unique Ergodicity of Automorphism Groups
}

\author{
Omer Angel, Alexander S. Kechris, and Russell Lyons ${ }^{\ddagger}$
}

9 August 2012

\begin{abstract}
We show that the only random orderings of finite graphs that are invariant under isomorphism and induced subgraph are the uniform random orderings. We show how this implies the unique ergodicity of the automorphism group of the random graph. We give similar theorems for other structures, including, for example, metric spaces. These give the first examples of uniquely ergodic groups, other than compact groups and extremely amenable groups, after Glasner and Weiss's example of the group of all permutations of the integers. We also contrast these results to those for certain special classes of graphs and metric spaces in which such random orderings can be found that are not uniform.
\end{abstract}

\section{Introduction}

\subsection{Random Orderings}

Consider the class of finite graphs, by which we mean simple graphs, i.e., without loops or multiple edges. Is there any way to distinguish among the vertices of a finite graph in a way that is preserved by isomorphism and by taking induced subgraphs? To make this question more precise, consider random linear (total) orderings of vertices of finite graphs. That is, for each graph $G=\langle V, E\rangle$, let $\mu_{G}$ be a probability measure on the $|V|$ ! linear orderings of $V$. Suppose that the collection of measures $\mu_{G}$ is consistent, meaning that it satisfies two properties:

i) If $\phi: G \rightarrow G^{\prime}$ is a graph isomorphism, then $\phi_{*} \mu_{G}=\mu_{G^{\prime}}$. Here, $\phi_{*}$ denotes the pushforward map induced by $\phi$; more precisely, $\phi_{*}$ is the push-forward of the map $(\phi, \phi)$ on orders.

\footnotetext{
*Research partially supported by NSERC and the Sloan Foundation

${ }^{\dagger}$ Research partially supported by NSF grant DMS-0968710.

${ }^{\ddagger}$ Research partially supported by NSF grant DMS-1007244 and Microsoft Research.
} 
ii) If $H$ is an induced subgraph of $G$, then $\mu_{G}$ induces $\mu_{H}$ by restriction. In other words, if $\phi$ is the restriction map of orderings of $V(G)$ to orderings of $V(H)$, then $\phi_{*} \mu_{G}=\mu_{H}$.

We shall refer to the family $\left(\mu_{G}\right)_{G}$ as a consistent random ordering (for the class of finite graphs). Note that property (i) by itself guarantees that for a complete graph $G$, as well as an empty graph, $\mu_{G}$ must be the uniform measure. If $\left(\mu_{G}\right)_{G}$ is consistent, must $\mu_{G}$ be the uniform measure on all linear orderings of $V(G)$ for all $G$, or is there a more interesting consistent assignment of random orderings?

For example, if instead of the collection of all finite graphs, we considered consistent random orderings only of finite graphs that are paths, then there is clearly another choice: orient the path in one of the two ways at random, with probability $1 / 2$ each, and use the orientation to define the naturally associated ordering.

It is much harder to define a non-uniform consistent random ordering on the class of finite graphs all of whose components are paths, but it can be done. What about graphs with a given bound on their degrees, or other classes of graphs? What about finite hypergraphs or finite metric spaces? In fact, such questions can be asked in great generality for classes of finite structures in a given language in the sense of model theory.

We show in this paper the following.

Theorem 1.1 The only consistent random ordering for the class of finite graphs is the uniform ordering. The same holds for the classes of $K_{n}$-free graphs, $r$-uniform hypergraphs, and metric spaces with (non-zero) distances in a given additive subsemigroup of $\mathbb{R}^{+}$.

This is proved in Sections 2-7, where many other such examples are given, including classes of hypergraphs with forbidden configurations. In these sections, we also discuss several examples of classes of metric spaces and graphs for which the opposite happens, i.e., there are non-uniform consistent random orderings, including the class of Euclidean metric spaces and the class of bounded degree graphs, which can be proved by using a random projection method suggested to us by Leonard Schulman.

Furthermore, we obtain quantitative versions of Theorem 1.1. Let $d_{\mathrm{TV}}(\mu, \nu):=\frac{1}{2} \| \mu-$ $\nu \|_{1}=\max _{A}|\mu(A)-\nu(A)|$ denote the total variation distance between probability measures. We show the following:

Theorem 1.2 Let $2 \leq r \leq k \leq n$ be integers. There is a constant $C=C(k, r)$ with the following property. Let $\left(\mu_{G}\right)_{G}$ be a consistent random ordering defined on all $r$-uniform hypergraphs $G$ of size at most $n$, and let $H$ be a r-uniform hypergraph of size $k$. If $\nu_{H}$ is the uniform ordering on $H$, then $d_{\mathrm{TV}}\left(\mu_{H}, \nu_{H}\right) \leq C \sqrt{\frac{\log n}{n^{r-1}}}$.

In the case of graphs, we construct in Section 3 a random ordering on graphs of size at most $n$ (or even with degrees bounded by $n$ ) such that for some $H, d_{\mathrm{TV}}\left(\mu_{H}, \nu_{H}\right) \geq C / n$. A similar construction appears to give $d_{\mathrm{TV}}\left(\mu_{H}, \nu_{H}\right) \geq C / n^{r-1}$ for $r$-uniform hypergraphs.

Question 1.3 What is the largest possible total variation distance from the uniform ordering of a random ordering on graphs (or hypergraphs) of size at most $n$ ? 


\subsection{Unique Ergodicity}

The reason for our attention to these questions, beyond their intrinsic interest, is that, in certain circumstances, they provide a way to prove unique ergodicity results for groups. In order to explain this context, we need to review some concepts and results concerning the model theory and combinatorics of classes of finite structures.

The general setting for our ergodicity results is the following. A (first-order) language $L$ consists of families $\left(R_{i}\right)_{i \in I},\left(f_{j}\right)_{j \in J}$ of relation symbols $R_{i}$ and function symbols $f_{j}$ with associated arities $m_{i} \geq 1$ and $n_{j} \geq 0$. A structure for this language, or L-structure, is an object of the form

$$
\boldsymbol{A}=\left\langle A,\left(R_{i}^{\boldsymbol{A}}\right)_{i \in I},\left(f_{j}^{\boldsymbol{A}}\right)_{j \in J}\right\rangle,
$$

where $A$ is a non-empty set, called the universe of the structure, $R_{i}^{\boldsymbol{A}} \subseteq A^{m_{i}}$ and $f_{j}^{\boldsymbol{A}}: A^{n_{j}} \rightarrow$ $A$, where if $n_{j}=0$, it is understood that $f_{j}^{\boldsymbol{A}}$ is just an element of $A$. The cardinality of the structure is the cardinality of its universe $A$. In this paper, all languages and structures will be countable. For brevity, and when there is no danger of confusion, we sometimes omit the superscripts.

As an example, if $L=\{E\}$ with $E$ a binary relation symbol, then the class of graphs is the class of $L$-structures $\boldsymbol{A}$ for which $E^{\boldsymbol{A}}$ is symmetric and irreflexive. A metric space $\langle X, d\rangle$ can also be viewed as a structure $\boldsymbol{X}=\left\langle X,\left(R_{q}^{\boldsymbol{X}}\right)_{q \in \mathbb{Q}^{+}}\right\rangle$in the language with binary relation symbols $\left(R_{q}\right)_{q \in \mathbb{Q}^{+}}$, where $R_{q}^{\boldsymbol{X}}(x, y) \Longleftrightarrow d(x, y)<q$.

Although this will be our standard notation when we discuss abstract structures, we shall keep the more traditional notation (mainly in font type) for specific structures, like graphs, hypergraphs, metric spaces, etc., that we used earlier and that we also use in Sections 2-7.

A class $\mathcal{K}$ of finite $L$-structures is called a Fraïssé class if it contains structures of arbitrarily large (finite) cardinality, is countable (in the sense that it contains only countably many isomorphism types) and satisfies the following:

i) Hereditary Property: If $\boldsymbol{B} \in \mathcal{K}$ and $\boldsymbol{A}$ can be embedded in $\boldsymbol{B}$, then $\boldsymbol{A} \in \mathcal{K}$.

ii) Joint Embedding Property: If $\boldsymbol{A}, \boldsymbol{B} \in \mathcal{K}$, there is $\boldsymbol{C} \in \mathcal{K}$ such that $\boldsymbol{A}, \boldsymbol{B}$ can be embedded in $\boldsymbol{C}$.

iii) Amalgamation Property: If $\boldsymbol{A}, \boldsymbol{B}, \boldsymbol{C} \in \mathcal{K}$ and $f: \boldsymbol{A} \rightarrow \boldsymbol{B}, g: \boldsymbol{A} \rightarrow \boldsymbol{C}$ are embeddings, there is $\boldsymbol{D} \in \mathcal{K}$ and embeddings $r: \boldsymbol{B} \rightarrow \boldsymbol{D}, s: \boldsymbol{C} \rightarrow \boldsymbol{D}$ such that $r \circ f=s \circ g$.

Throughout this paper embeddings and substructures will be understood in the usual model theoretic sense (see, e.g., Hodges [Ho], page 5); e.g., for graphs embeddings are induced embeddings, i.e., isomorphisms onto induced subgraphs. To be precise, given a language $L$ consisting of families $\left(R_{i}\right)_{i \in I}$ and $\left(f_{j}\right)_{j \in J}$ of relation symbols $R_{i}$ and function symbols $f_{j}$ with associated arities $m_{i} \geq 1$ and $n_{j} \geq 0$, an embedding of an L-structure $\boldsymbol{A}$ into an $L$-structure $\boldsymbol{B}$ is an injection $\phi: A \rightarrow B$ such that for any $R_{i}$ and $a_{1}, \ldots, a_{m_{i}} \in A$, we have $R_{i}^{\boldsymbol{A}}\left(a_{1}, \ldots, a_{m_{i}}\right) \Longleftrightarrow R_{i}^{\boldsymbol{B}}\left(\phi\left(a_{1}\right), \ldots, \phi\left(a_{m_{i}}\right)\right)$ and for any $f_{j}$ and any $a_{1}, \ldots, a_{n_{j}} \in A$, we have $\phi\left(f_{j}^{\boldsymbol{A}}\left(a_{1}, \ldots, a_{n_{j}}\right)\right)=f_{j}^{\boldsymbol{B}}\left(\phi\left(a_{1}\right), \ldots, \phi\left(a_{n_{j}}\right)\right)$. If the identity is such an embedding, then we say that $\boldsymbol{A}$ is a substructure of $\boldsymbol{B}$. 
We recall the following results of $[\mathrm{Fr}]$ (see also [Ho, Section 7.1]):

If $\mathcal{K}$ is a Fraïssé class, there is a unique, up to isomorphism, countably infinite structure $\boldsymbol{K}$ that is locally finite (i.e., each finite subset of $K$ is contained in a finite substructure of $\boldsymbol{K}$ ), ultrahomogeneous (i.e., any isomorphism between finite substructures can be extended to an automorphism of the structure) and is such that, up to isomorphism, its finite substructures are exactly those in $\mathcal{K}$. We call this the Fraïssé limit of $\mathcal{K}$, in symbols

$$
\boldsymbol{K}=\mathrm{F} \lim (\mathcal{K})
$$

Conversely, if $\boldsymbol{K}$ is a countably infinite structure that is locally finite and ultrahomogeneous, then its age, $\operatorname{Age}(\boldsymbol{K})$, i.e., the class $\mathcal{K}$ of all its finite substructures, up to isomorphism, is a Fraïssé class. Such structures $\boldsymbol{K}$ are called Frä̈ssé structures. Thus there is a canonical bijection $\mathcal{K} \mapsto \operatorname{Flim}(\mathcal{K}), \boldsymbol{K} \mapsto \operatorname{Age}(\boldsymbol{K})$ between Fraïssé classes and structures.

We are interested in the set of invariant Borel probability measures for continuous actions of the automorphism group $\Gamma:=\operatorname{Aut}(\boldsymbol{K})$, viewed as a topological group under the pointwise convergence topology. We note that the groups $\operatorname{Aut}(\boldsymbol{K})$, for Fraïssé structures $\boldsymbol{K}$ as above, are (up to topological group isomorphism) exactly the closed subgroups of the infinite symmetric group $S_{\infty}$, i.e, the group of permutations of $\mathbb{N}$, again with the pointwise convergence topology (see, e.g., [KPT]). Up to topological group isomorphism they are also the same as the non-archimedean Polish groups, where a topological group is called non-archimedean if it admits a basis at the identity consisting of open subgroups (see [BK, 1.5.1]).

Consider now amenability properties of such groups. At one end of the spectrum, there are many examples of automorphism groups that satisfy a very strong form of amenability, i.e., they are extremely amenable. This means that every continuous action of such a group on a (non-empty) compact Hausdorff space, i.e., a $\Gamma$-flow, has a fixed point. As was shown in [KPT, 4.7], $\Gamma=\operatorname{Aut}(\boldsymbol{K})$ has this property exactly when $\boldsymbol{K}$ is an order Fraïssé structure, i.e., a Fraïssé structure in which one of the relations is a linear ordering, such that $\operatorname{Age}(\boldsymbol{K})$ satisfies the Ramsey Property (RP); see [KPT, Section 3]. We also discuss the RP in Section 9 below. Extensive lists of extremely amenable automorphism groups are discussed in [KPT, Section 6].

Next there are automorphism groups that are amenable (i.e., every $\Gamma$-flow has an invariant Borel probability measure) but not extremely amenable. The most extensive list, in our framework, of such examples arises in the context of the Hrushovski property. Given a class $\mathcal{K}$ of finite structures in a given language, we say that $\mathcal{K}$ is a Hrushovski class if for any $\boldsymbol{A} \in \mathcal{K}$ and any (partial) isomorphisms $\varphi_{i}: \boldsymbol{B}_{i} \rightarrow \boldsymbol{C}_{i}, 1 \leq i \leq k$, where $\boldsymbol{B}_{i}, \boldsymbol{C}_{i}$ are substructures of $\boldsymbol{A}$, there is $\boldsymbol{B} \in \mathcal{K}$ containing $\boldsymbol{A}$ as a substructure such that each $\varphi_{i}$ can be extended to an automorphism $\psi_{i}$ of $\boldsymbol{B}, 1 \leq i \leq k$. It is shown in $[\mathrm{KR}, 6.4]$ that if $\mathcal{K}$ is a Fraïssé class with $\boldsymbol{K}=\mathrm{Flim}(\mathcal{K})$, then $\mathcal{K}$ is a Hrushovski class iff $\Gamma=\operatorname{Aut}(\boldsymbol{K})$ is compactly approximable, i.e., there is an increasing sequence $\Delta_{0} \subseteq \Delta_{1} \subseteq \cdots$ of compact subgroups of $\Gamma$ with $\overline{\bigcup_{n} \Delta_{n}}=\Gamma$. Calling the Fraïssé limit $\boldsymbol{K}$ of a Hrushovski class $\mathcal{K}$ a $\boldsymbol{H r u s h o v s k i}$ structure, we thus see that the automorphism groups of Hrushovski structures are compactly approximable, whence amenable. Examples of Hrushovski classes of finite structures include the following: pure sets, graphs (Hrushovski [Hr]), $r$-uniform hypergraphs, $K_{n}$-free 
graphs (Herwig [He1, He2]), metric spaces with distances in a countable additive subsemigroup of $\mathbb{R}^{+}$(Solecki $[\mathrm{So}]$ ), finite-dimensional vector spaces over a fixed finite field, etc.

Finally, at the other end of the spectrum, there are also automorphism groups $\Gamma=$ $\operatorname{Aut}(\boldsymbol{K})$ that are not amenable. These include, among others, the automorphism groups of the countable atomless Boolean algebra, the random poset, and the random distributive lattice (see $[K S])$.

A characterization of the amenability of $\Gamma=\operatorname{Aut}(\boldsymbol{K})$ in terms of combinatorial properties of the Fraïssé class $\operatorname{Age}(\boldsymbol{K})$ was obtained by Moore and Tsankov, see [Mo, 6.1]. Another characterization, in a special case, is in Proposition 9.2 below.

In this paper we are interested in the ergodic theory of the flows of automorphism groups, in particular, in the potential phenomenon of unique ergodicity. Below, measure means Borel probability measure.

Let $\Gamma$ be a topological group. We say that a $\Gamma$-flow is uniquely ergodic if it has a unique invariant measure (which therefore must be ergodic). We say that the group $\Gamma$ is uniquely ergodic if every minimal $\Gamma$-flow (i.e., one all of whose orbits are dense) is uniquely ergodic. (The assumption of minimality is clearly necessary, as a given $\Gamma$-flow may have in general many minimal subflows that are pairwise disjoint, and by amenability each will support an invariant measure.) Clearly every extremely amenable Polish group is uniquely ergodic and so is every compact Polish group.

However, this property is never realized in the realm of infinite countable (discrete) groups, as follows from results of Benjamin Weiss [W]. Weiss also believes that this extends to non-compact, Polish locally compact groups, although this has not been checked in detail yet.

It is important here to review the concept of the universal minimal flow of a topological group, $\Gamma$. It is a classical result in topological dynamics that every topological group $\Gamma$ admits a unique, up to isomorphism of $\Gamma$-flows, minimal flow $M(\Gamma)$, called its universal minimal flow, such that all other minimal $\Gamma$-flows are factors of it (see, e.g., [KPT, Section 1]). Recall that a $\Gamma$-flow $Y$ is a factor of a $\Gamma$-flow $X$ if there is a continuous surjection $\phi: X \rightarrow Y$ that is a $\Gamma$-map, i.e., $\phi(\gamma \cdot x)=\gamma \cdot \phi(x)$ for every $x \in X$ and $\gamma \in \Gamma$. If such a map is a bijection, we call it an isomorphism. Since every $\Gamma$-flow contains a minimal subflow, the group $\Gamma$ is amenable iff $M(\Gamma)$ has an invariant measure. Also, it can be seen that $\Gamma$ is uniquely ergodic iff $M(\Gamma)$ is uniquely ergodic (see Proposition 8.1).

When $\Gamma$ is compact, $M(\Gamma)=\Gamma$ (with the left translation action) and when $\Gamma$ is extremely amenable, $M(\Gamma)$ is trivial, i.e., a singleton, but in general $M(\Gamma)$ is a very complicated object that is difficult to "compute". For example, when $\Gamma$ is infinite countable, $M(\Gamma)$ is a "big" space of ultrafilters on $\Gamma$, and more generally when $\Gamma$ is non-compact, locally compact, $M(\Gamma)$ is not metrizable (see [KPT, A2.2]). However, over the last fifteen years or so, there have been many examples of explicit descriptions of non-trivial metrizable universal minimal flows: see Pestov [Pe1], Glasner and Weiss [GW1, GW2] and, in the case of automorphism groups of Fraïssé structures, [KPT].

As was shown in [GW1], where the universal minimal flow of $S_{\infty}$ was computed, $S_{\infty}$ is uniquely ergodic. This seems to be the first example found of such a group that is neither 
extremely amenable nor compact. We shall show in this paper that many other amenable automorphism groups, for which we can compute a metrizable universal minimal flow, are also uniquely ergodic, so this appears as a general phenomenon. This will be a consequence of the uniqueness results for consistent random orderings, like Theorem 1.1, that we prove in Sections 2-6.

To see this connection, we need to explain briefly the method by which universal minimal flows are calculated in [KPT]. Details and precise definitions are given in Section 9. Given a Fraïssé class $\mathcal{K}$ with Fraïssé limit $\boldsymbol{K}$, one appropriately assigns to each structure $\boldsymbol{A} \in \mathcal{K}$ a collection of so-called admissible (linear) orderings on the universe $A$ of $\boldsymbol{A}$ to obtain a new class $\mathcal{K}^{*}$ of expanded, ordered structures of the form $\langle\boldsymbol{A},<\rangle$, where $\boldsymbol{A} \in \mathcal{K}$ and $<$ is an admissible ordering on $\boldsymbol{A}$. In many cases, such as for graphs, every linear ordering on a given structure is admissible, but in other cases, one has to take a more restricted collection of linear orderings. For example, take as $\mathcal{K}$ the Fraïssé class of bipartite graphs with distinguished parts, i.e., structures of the form $\left\langle V, E, A_{1}, A_{2}\right\rangle$, where $\langle V, E\rangle$ is a graph and $A_{1}, A_{2}$ is a partition of $V$ such that there are no edges within each $A_{i}$. Then for each such graph, the relevant collection of admissible orderings consists of all orderings $<$ such that $A_{1}<A_{2}$.

If an appropriate such class $\mathcal{K}^{*}$ of expanded structures as above can be found that satisfies various structural conditions, including Ramsey properties, we shall call $\mathcal{K}^{*}$ a "companion" of $\mathcal{K}$. In that case, it is shown in [KPT] that the universal minimal flow of $\Gamma=\operatorname{Aut}(\boldsymbol{K})$ is obtained as follows. Denote by $X_{\mathcal{K}^{*}}$ the space of all orderings $<$ on the universe $K$ of $\boldsymbol{K}$ that are admissible (relative to $\mathcal{K}^{*}$ ) in the sense that for every finite substructure $\boldsymbol{A}$ of $\boldsymbol{K}$, the restriction of $<$ to $A$ is admissible (for $\boldsymbol{A}$ ). This is a compact metrizable space on which $\Gamma$ acts continuously in the obvious way, and it turns out to be the universal minimal flow for $\Gamma$. From this one can see that the existence of an invariant measure on $X_{\mathcal{K}^{*}}$ (i.e., the amenability of $\Gamma$ ) is equivalent to the existence of a consistent random admissible ordering and unique ergodicity of $\Gamma$ is equivalent to the uniqueness of a consistent random admissible ordering. Using this and the results in Sections 2-6 concerning uniqueness of consistent random orderings, we obtain, in Sections 10-11, many new examples of uniquely ergodic automorphism groups. A sample is included in the following theorem, which we state after we introduce some terminology.

If $L=\left(R_{i}\right)_{i \in I}$ is a finite relational language with $R_{i}$ of arity $m_{i} \geq 2$, then a hypergraph of type $L$ is an $L$-structure $\boldsymbol{A}$ in which each $R_{i}^{\boldsymbol{A}}$ gives an $m_{i}$-uniform hypergraph. A hypergraph of type $L$ is called irreducible if it has at least two vertices and every twoelement subset of the vertices belongs to some hyperedge. Given a class $\mathcal{A}$ of irreducible hypergraphs of type $L$, a hypergraph of type $L$ is $\mathcal{A}$-free if it contains no (induced) copy of a structure in $\mathcal{A}$. If we choose $L$ to have only one relation symbol of arity $r$ and $\mathcal{A}=\emptyset$, then we obtain the class of $r$-uniform hypergraphs (graphs if $r=2$ ), and if we choose $\mathcal{A}=\emptyset$, we obtain the class of all hypergraphs of type $L$. If we choose $L$ to have only one symbol of arity 2 and $\boldsymbol{A}=\left\{K_{n}\right\}$, then we obtain the class of $K_{n}$-free graphs, where $K_{n}$ is the complete graph on $n$ vertices.

The random $\mathcal{A}$-free hypergraph of a given type $L$ is the Fraïssé limit of the class of 
$\mathcal{A}$-free hypergraphs of type $L$ (thus by choosing $L$ and $\mathcal{A}$ appropriately, this includes the case of the random graph, random $K_{n}$-free graph, random $r$-uniform hypergraph, etc.). The Urysohn space $\boldsymbol{U}_{S}$, where $S$ is a countable additive subsemigroup of $\mathbb{R}^{+}$, is the Fraïssé limit of the class of finite metric spaces with distances in $S$.

Theorem 1.4 The automorphism groups of the following structures are uniquely ergodic: the (countably) infinite-dimensional vector space over a given finite field, the random $\mathcal{A}$-free hypergraph of a given type, and $\boldsymbol{U}_{S}$.

Restricting ourselves to automorphism groups of Hrushovski structures, which provide the most prominent examples of amenable (but not extremely amenable) groups, we shall find that unique ergodicity of $\operatorname{Aut}(\boldsymbol{K})$, with $\boldsymbol{K}$ a Hrushovski structure, is equivalent to a combinatorial property of $\operatorname{Age}(\boldsymbol{K})$, very much in the spirit of [KPT]. In fact, rather interestingly, if $\mathcal{K}:=\operatorname{Age}(\boldsymbol{K})$ admits a companion $\mathcal{K}^{*}$ as above, then it turns out that unique ergodicity is exactly equivalent to a quantitative version of what is called the "ordering property". The ordering property is a key ingredient of the Ramsey theory of classes of finite structures that is instrumental in the computation of universal minimal flows in [KPT, 7.5]. We discuss this in Section 13 below.

In Section 14, we show that for certain automorphism groups, including those of the random $\mathcal{A}$-free uniform hypergraph of a given type and of $\boldsymbol{U}_{S}$, every minimal action not only has a unique invariant measure, but also this measure concentrates on a single comeager orbit. This was earlier proved for the group $S_{\infty}$ by Glasner and Weiss [GW1].

Finally in the last Section 15, we discuss some open problems arising from the work in this paper.

\section{Graphs and Uniform Hypergraphs}

In this section, we prove that the only consistent random ordering on the class of all finite graphs is the uniform ordering. In fact, we prove the same for hypergraphs. Recall that an r-uniform hypergraph is a pair $G=\langle V, E\rangle$, where $E \subseteq\left(\begin{array}{l}V \\ r\end{array}\right)$ is a collection of subsets of $V$ of cardinality $r$; the elements of $E$ are called hyperedges. The case $r=2$ is the case of graphs. The size of $G$ is defined to be the cardinality of $V$. If $G=\langle V, E\rangle$ is a hypergraph and $V^{\prime} \subseteq V$, then the hypergraph induced on $V^{\prime}$ by $G$ equals $\left\langle V^{\prime}, E \cap\left(\begin{array}{c}V^{\prime} \\ r\end{array}\right)\right\rangle$. Note that hyperedges intersecting $V^{\prime}$ that are not contained in $V^{\prime}$ are discarded.

The way we prove Theorem 1.2 is via the following general principle. Let $N_{\text {ind }}(H, G)$ denote the number of embeddings of $H$ in $G$, i.e., the number of isomorphisms $\phi: H \rightarrow H^{\prime}$ such that $H^{\prime}$ is an induced hypergraph in $G$. (Up to symmetries, this is the number of induced subgraphs of $G$ that are isomorphic to $H$.) Given a pair of orderings $<_{G}$ of $V(G)$ and $<_{H}$ of $V(H)$, let $N_{\text {ord }}(H, G)$ denote the number of ordered embeddings of $H$ in $G$, i.e., the number of embeddings $\phi: H \rightarrow G$ such that $\phi^{*}\left(<_{G}\right)=<_{H}$. Here, $\phi^{*}$ denotes the pull-back map induced by $\phi$, i.e., $x<_{\phi^{*}\left(<_{G}\right)} y \Longleftrightarrow \phi(x)<_{G} \phi(y)$. 
Lemma 2.1 Let $k \geq r \geq 2$ be integers. Let $G$ be an $r$-uniform hypergraph and $H$ be an $r$-uniform hypergraph on $k$ vertices such that $N_{\text {ind }}(H, G)>0$. Suppose $\delta$ is such that for every pair of orderings $<_{G}$ of $V(G)$ and $<_{H}$ of $V(H)$,

$$
\left|\frac{N_{\text {ord }}(H, G)}{N_{\text {ind }}(H, G)}-\frac{1}{k !}\right| \leq \delta \text {. }
$$

Let $\mu_{G}$ and $\mu_{H}$ be random orderings on $G$ and $H$, respectively. Suppose that every embedding $\phi$ of $H$ in $G$ satisfies $\phi^{*} \mu_{G}=\mu_{H}$. Then $d_{\mathrm{TV}}\left(\mu_{H}, \nu_{H}\right) \leq \delta k ! / 2$, where $\nu_{H}$ is the uniform ordering on $H$.

Proof. Fix $<_{H}$. Choose $<_{G}$ at random according to $\mu_{G}$ and choose an embedding $\boldsymbol{\Phi}$ of $H$ in $G$ uniformly at random. Let $A$ be the event that the restriction of $<_{G}$ to the image of $\boldsymbol{\Phi}$ equals $\boldsymbol{\Phi}_{*}\left(<_{H}\right)$. Since $\phi^{*} \mu_{G}=\mu_{H}$ for every $\phi$, we have $\mathbf{P}(A \mid \boldsymbol{\Phi}=\phi)=\mu_{H}\left(<_{H}\right)$, whence averaging over $\phi$ gives $\mathbf{P}(A)=\mu_{H}\left(<_{H}\right)$. We can rewrite the assumption (1) as $\left|\mathbf{P}\left(A \mid<_{G}=<_{G}\right)-1 / k !\right| \leq \delta$ for each $<_{G}$; averaging over $<_{G}$ gives $|\mathbf{P}(A)-1 / k !| \leq \delta$. That is, $\left|\mu_{H}\left(<_{H}\right)-1 / k !\right| \leq \delta$. Finally, summing over all orderings $<_{H}$ gives the bound.

Clearly Theorem 1.2 follows from Lemma 2.1 and the following result. Write $I(n, k, r):=$ $(n)_{k} 2^{-\left(\begin{array}{c}k \\ r\end{array}\right)}$, where $(n)_{k}:=n(n-1) \ldots(n-k+1)$ is the number of $1-1$ maps from $\{1, \ldots, k\}$ to $\{1, \ldots, n\}$.

Theorem 2.2 Let $2 \leq r \leq k \leq n$ be integers. There is a constant $C=C(k, r)$ with the following property. For every $r$-uniform hypergraph $H$ on $k$ vertices, there exists an $r$-uniform hypergraph $G$ on $n$ vertices such that

$$
\left|\frac{N_{\text {ind }}(H, G)}{I(n, k, r)}-1\right|<C \sqrt{\frac{\log n}{n^{r-1}}}
$$

and for every pair of orderings $<_{G}$ of $V(G)$ and $<_{H}$ of $V(H)$,

$$
\left|\frac{N_{\text {ord }}(H, G)}{N_{\text {ind }}(H, G)}-\frac{1}{k !}\right|<C \sqrt{\frac{\log n}{n^{r-1}}} .
$$

The proof of Theorem 2.2 uses the following classical inequality of McDiarmid [McD], known as the bounded-differences inequality:

Theorem 2.3 Let $\mathbf{Z}:=\left\langle Z_{1}, \ldots, Z_{n}\right\rangle$, where $Z_{i}$ are independent random variables, and $f\left(z_{1}, \ldots, z_{n}\right)$ be a real-valued function such that

$$
\left|f(\mathbf{z})-f\left(\mathbf{z}^{\prime}\right)\right| \leq a_{i}
$$

when the vectors $\mathbf{z}$ and $\mathbf{z}^{\prime}$ differ only in the ith coordinate. Write $\zeta:=\mathbf{E}[f(\mathbf{Z})]$. Then for all $L>0$,

$$
\mathbf{P}[|f(\mathbf{Z})-\zeta| \geq L] \leq 2 \exp \left(-\frac{2 L^{2}}{\sum_{i=1}^{n} a_{i}^{2}}\right) .
$$


Proof of Theorem 2.2. Let $\mathbf{G}$ be a uniformly random $r$-uniform hypergraph on $n$ fixed vertices, $V$ (so that each hyperedge is present with probability $1 / 2$ ). Note that

$$
I(n, k, r)=\mathbf{E}\left[N_{\text {ind }}(H, \mathbf{G})\right] .
$$

Define

$$
f(\mathbf{G}):=\frac{N_{\text {ind }}(H, \mathbf{G})}{I(n, k, r)},
$$

which we consider as a function of the $\left(\begin{array}{l}n \\ r\end{array}\right)$ variables indicating the presence of each possible hyperedge. The addition or removal a single hyperedge to $\mathbf{G}$ changes $N_{\text {ind }}(H, \mathbf{G})$ by at most $(k)_{r}(n-r)_{k-r}$, and so $f$ satisfies the conditions of Theorem 2.3 with $a_{i}=c_{1} n^{-r}$, where we shall denote by $c_{j}$ intermediate constants that depend on $k$ and $r$, but not on $n$. It follows that

$$
\mathbf{P}\left[\left|\frac{N_{\text {ind }}(H, \mathbf{G})}{I(n, k, r)}-1\right| \geq D\right] \leq 2 \exp \left\{\frac{-2 D^{2}}{\left(\begin{array}{l}
n \\
r
\end{array}\right)\left(c_{1} n^{-r}\right)^{2}}\right\} \leq 2 e^{-c_{2} D^{2} n^{r}} .
$$

Similarly, for any fixed orderings $<_{H}$ and $<_{V}$, we have $\mathbf{E}\left[N_{\text {ord }}(H, \mathbf{G})\right]=I(n, k, r) / k$ !. We apply Theorem 2.3 to

$$
\mathbf{G} \mapsto \frac{N_{\text {ord }}(H, \mathbf{G})}{I(n, k, r)}
$$

Here, adding or removing a single hyperedge changes $N_{\text {ord }}(H, \mathbf{G})$ by at most $\left(\begin{array}{l}k \\ r\end{array}\right)(n-r)_{k-r}$, so as above,

$$
\mathbf{P}\left[\left|\frac{N_{\text {ord }}(H, \mathbf{G})}{I(n, k, r)}-\frac{1}{k !}\right| \geq D\right] \leq 2 \exp \left\{\frac{-2 D^{2}}{\left(\begin{array}{l}
n \\
r
\end{array}\right)\left(c_{3} n^{-r}\right)^{2}}\right\} \leq 2 e^{-c_{4} D^{2} n^{r}} .
$$

Combining these, we find that except with probability $c_{5} n ! e^{-c_{6} D^{2} n^{r}}$, we have simultaneously

$$
\left|\frac{N_{\text {ind }}(H, \mathbf{G})}{I(n, k, r)}-1\right|<D \quad \text { and } \quad\left|\frac{N_{\text {ord }}(H, \mathbf{G})}{I(n, k, r)}-\frac{1}{k !}\right|<D
$$

for all orderings $<_{H}$ and $<_{V}$. We now take $D:=c_{7} \sqrt{\frac{\log n}{n^{r-1}}}$ with $c_{7}$ chosen so that $c_{5} n ! e^{-c_{6} D^{2} n^{r}}<$ 1. Then there is a $G$ satisfying the above bounds. The claim then follows by the triangle inequality with $C:=2 c_{7}$, since

$$
\left|\frac{N_{\text {ord }}(H, G)}{I(n, k, r)}-\frac{N_{\text {ord }}(H, G)}{N_{\text {ind }}(H, G)}\right|=\frac{N_{\text {ord }}(H, G)}{N_{\text {ind }}(H, G)} \cdot\left|\frac{N_{\text {ind }}(H, G)}{I(n, k, r)}-1\right|<1 \cdot D .
$$

This method of proof can be applied to many other classes of structures, thereby establishing the uniqueness of consistent random (admissible) orderings for these classes. These include: (i) the Fraïssé class of finite tournaments and (ii) the Fraïssé class of arbitrary $L$-structures for any finite language $L$ containing only relation symbols of arity $\geq 2$. In both these cases, the uniform ordering is the unique consistent random ordering. For another 
example, consider a finite language $L$ containing at least one relation symbol of arity $\geq 2$ and unary relation symbols $P_{1}, \ldots, P_{k}$ and consider the Fraïssé class of structures for this language in which the $P_{1}, \ldots, P_{k}$ form a partition. In this case, the admissible orderings for such a structure $\boldsymbol{A}$ will turn out to be those $<$ for which $P_{1}^{\boldsymbol{A}}<\cdots<P_{k}^{\boldsymbol{A}}$ and again the uniform ordering is the unique consistent random admissible ordering. (This also holds if the language contains no relation symbols of arity $\geq 2$, but uniqueness is straightforward in this case and does not need the methods of this section; see Section 10.) Similarly, take as $\mathcal{K}$ the Fraïssé class of bipartite graphs with distinguished parts, i.e., structures of the form $\left\langle V, E, A_{1}, A_{2}\right\rangle$, where $\langle V, E\rangle$ is a graph and $A_{1}, A_{2}$ is a partition of $V$ such that there are no edges within each $A_{i}$. Then for each such graph the relevant collection of admissible orderings consists of all orderings $<$ such that $A_{1}<A_{2}$ and the uniform ordering is the unique consistent random admissible ordering.

\section{Bounded Degree Graphs (and Hypergraphs?)}

In this section, we construct non-uniform consistent random orderings on graphs with bounded degrees, and estimate their total variation distance from uniform. We believe we have a construction for hypergraphs as well, but lack a proof.

Theorem 3.1 There is a constant $C>0$ with the following property. Let $n \geq 2$ be an integer. There is a consistent random ordering $\left(\mu_{G}\right)_{G}$ defined on all graphs of size at most $n$ such that for every $k \in[3, n]$, there exists a graph $H$ of size $k$ with $d_{\mathrm{TV}}\left(\mu_{H}, \nu_{H}\right) \geq C / n$.

In fact, we prove the following more general lower bound.

Theorem 3.2 There is a constant $C>0$ with the following property. Let $D \geq 2$ be an integer. There exists a consistent random ordering $\left(\mu_{G}\right)_{G}$ defined on all graphs of degree at most $D$ such that for every $k \geq 3$, there exists an graph $H$ of degree at most $D$ and of size $k$ such that $d_{\mathrm{TV}}\left(\mu_{H}, \nu_{H}\right) \geq C / D$.

Proof. Let $G=\langle V, E\rangle$ be a graph with maximal degree $D$. Let us make every vertex $x \in V$ have degree exactly $D$ by adding $D-d_{x}$ additional edges connecting $x$ to new, auxiliary vertices. Call the resulting graph $\widehat{G}$. Let $Z(e)$ be independent standard normal random variables for the edges $e$ of $\widehat{G}$. Define $Y(x):=\sum_{e \ni x} Z(e)$ for vertices $x$ of $G$. (We do not bother defining $Y$ for vertices of $\widehat{G} \backslash G$.) Assign to the vertices of $G$ the order induced from $Y \subset \mathbb{R}$.

If $H$ is an induced subgraph of $G$, then the inclusion of $H$ in $G$ can be extended to a map from $\widehat{H}$ to $\widehat{G}$ that is 1-1 on edges (though some of the vertices added to $H$ may be mapped to the same vertex of $G$.) Now the IID Gaussians associated with the edges of $\widehat{G}$ can be pulled back to $\widehat{H}$. This gives the ordering of $H$ as the restriction of the ordering of $G$, thus showing that this ordering is consistent.

However, this ordering is not uniform. Given $k$, let $H$ be the graph on vertices $\{1, \ldots, k\}$ with only two edges, $e_{1}:=(1,2)$ and $e_{2}:=(2,3)$. Let $A$ be the event that $Y(1)<Y(2)<$ 
$Y(3)$. Then $\nu_{H}(A)=1 / 6$, whereas $\mu_{H}(A) \geq 1 / 6+1 /(6 D)+o(1 / D)$. To see this latter fact, define $W_{i}:=Y(i)-Z\left(e_{1}\right)-Z\left(e_{2}\right)$, so that $A=\left\{W_{1}<W_{2}<W_{3}\right\}$. Note that $W_{1}, W_{2}$, and $W_{3}$ are independent normal random variables with variances $D, D-2$, and $D$, respectively. Therefore, $\mathbf{P}(A)=\mathbf{P}\left[Z_{1}<(1-2 / D) Z_{2}<Z_{3}\right]$ for independent standard normal random variables $Z_{1}, Z_{2}$, and $Z_{3}$. Define $f(\epsilon):=\mathbf{P}\left[Z_{1}<(1-\epsilon) Z_{2}<Z_{3}\right]$. It suffices to show that $f^{\prime}(0)=1 /(2 \pi \sqrt{3})>1 / 12$.

Write $\varphi(x)$ for the standard normal probability density. Then for $a<b$,

$$
\left.\frac{d}{d \epsilon} \mathbf{P}[a<(1-\epsilon) Z<b]\right|_{\epsilon=0}=b \varphi(b)-a \varphi(a),
$$

whereas for $a>b$ the derivative is trivially 0 . Thus

$$
\begin{aligned}
\left.\frac{d}{d \epsilon} \mathbf{P}\left[Z_{1}<(1-\epsilon) Z_{2}<Z_{3}\right]\right|_{\epsilon=0} & =\iint_{z_{3}>z_{1}}\left(z_{3} \varphi\left(z_{3}\right)-z_{1} \varphi\left(z_{1}\right)\right) \varphi\left(z_{1}\right) \varphi\left(z_{3}\right) d z_{1} d z_{3} \\
& =\frac{1}{2 \pi \sqrt{3}} .
\end{aligned}
$$

An even easier construction holds for orderings of finite connected bipartite graphs (such as trees) whose parts are not distinguished. There we have the following:

Theorem 3.3 There is a non-uniform consistent random ordering of the class of finite connected bipartite graphs.

Proof. We essentially saw this at the end of Section 2: Given a finite connected bipartite graph, let $A$ and $B$ be its two parts, named in random order. Order all of $A$ uniformly and all of $B$ uniformly independently, making all of $A$ less than all of $B$. It is easy to check that this is consistent.

Question 3.4 Is there a non-uniform consistent random ordering on all finite bipartite graphs? Is there a non-uniform consistent random ordering on finite forests? Is there a non-uniform consistent random ordering on finite planar graphs?

Remark 3.5 We believe that the following extension of Theorem 3.1 to uniform hypergraphs holds. Let $n>r \geq 2$ be integers. Then there is a constant $C(r)>0$ and a consistent random ordering $\left(\mu_{G}\right)_{G}$ defined on all $r$-uniform hypergraphs of size at most $n$ such that for every $k \in$ $[r+1, n]$, there exists an $r$-uniform hypergraph $H$ of size $k$ with $d_{\mathrm{TV}}\left(\mu_{H}, \nu_{H}\right) \geq C(r) / n^{r-1}$. In fact, we believe the following more general lower bound. Note that the degree of a vertex in a hypergraph is defined to be the number of hyperedges that contain the vertex. Let $r, D \geq 2$ be integers. Then there is a constant $C(r)>0$ with the following property: There exists a consistent random ordering $\left(\mu_{G}\right)_{G}$ defined on all $r$-uniform hypergraphs of degree at most $D$ such that for every $k \geq r+1$, there exists an $r$-uniform hypergraph $H$ of degree at most $D$ and of size $k$ such that $d_{\mathrm{TV}}\left(\mu_{H}, \nu_{H}\right) \geq C(r) / D$. 
It appears via simulations that the following modification of the proof of Theorem 3.1 should work. The consistency condition (ii) means that for $r \geq 3$, assigning a single Gaussian variable to each hyperedge and summing the variables of the edges containing a vertex does not yield a consistent ordering. The following construction is consistent and appears to give the claimed lower bound. Let $Z:=\left\langle Z_{1}, Z_{2}, \ldots, Z_{r}\right\rangle$ be a collection of $(r-1)$-independent exchangeable standard normal random variables that are not independent. (Recall that $Z$ is exchangeable means that the law of $Z$ is invariant under permutations of its coordinates.) For example, let $\Phi$ be the standard normal cumulative distribution function. Let $T_{1}, \ldots, T_{r}$ be IID uniform $[0,1]$ random variables conditioned to sum to $0 \bmod 1$. Now define $Z_{i}:=\Phi^{-1}\left(T_{i}\right)$. Let $G$ be an $r$-uniform hypergraph with maximal degree $D$. Let $Z(e)=\left\langle Z(e)_{x} ; x \in e\right\rangle$ be independent copies of $Z$ for the hyperedges $e$ of $G$. Also, for each vertex $x$ of $G$ of degree $d_{x}<D$, let $Z(x, 1), \ldots, Z\left(x, D-d_{x}\right)$ be additional independent standard normal random variables. Define $Y(x):=\sum_{e \ni x} Z(e)_{x}+\sum_{i=1}^{D-d_{x}} Z(x, i)$ for vertices $x$ of $G$. Note that the process $Y$ is $\operatorname{Aut}(G)$-invariant. Assign the vertices of $G$ the order induced from $Y \subset \mathbb{R}$. This is consistent and appears not to be uniform.

In fact, given $k$, let $H$ be the hypergraph consisting of the vertices $\left\{x_{1}, x_{2}, \ldots, x_{k}\right\}$ and two hyperedges, $e_{1}:=\left\{x_{1}, x_{2}, \ldots, x_{r}\right\}$ and $e_{2}:=\left\{x_{2}, x_{3}, \ldots, x_{r+1}\right\}$. Let $A$ be the event that $Y\left(x_{1}\right)<Y\left(x_{2}\right)<\cdots<Y\left(x_{r}\right)<Y\left(x_{r+1}\right)$. Then $\nu_{H}(A)=1 /(r+1)$ !, whereas it seems that $\mu_{H}(A) \geq 1 /(r+1) !+C(r) / D+o(1 / D)$.

\section{Dense Hypergraphs of Large Girth}

Here we prove a version of a lemma of [NR] that will be very useful to us in analyzing more complicated structures in the next two sections. There are various kinds of paths one can define in a hypergraph. We use the following. A path in a hypergraph is an alternating sequence $\left\langle x_{1}, e_{1}, x_{2}, e_{2}, \ldots, x_{L}, e_{L}, x_{L+1}\right\rangle$ of vertices $x_{i}$ and hyperedges $e_{i}$ such that $x_{i} \neq x_{i+1}$, $x_{i}, x_{i+1} \in e_{i}$, and $e_{i} \neq e_{i+1}$ for all $i \in[1, L]$. Such a path is said to join $x_{1}$ to $x_{L+1}$, to have length $L$, and to be a cycle if $L \geq 2$ and $x_{1}=x_{L+1}$. The girth of a hypergraph is the minimal length of a cycle that it contains. A hypergraph is connected if every pair of distinct vertices is joined by some path.

Lemma 4.1 Let $r \geq 2$ and $g \geq 3$ be integers. There is a constant $C=C(r, g)$ so that for all $n \geq r$, there exists an $r$-uniform hypergraph on $n$ vertices and at least $C n^{(g-1) /(g-2)}$ hyperedges that has girth at least $g$.

Proof. The (standard) method is to take a random hypergraph, and remove all edges that are in short cycles. Let $c_{i}$ denote constants that depend on $r$ and $g$, but not on $n$. Let $p:=a / n^{r-(g-1) /(g-2)}$ for a small constant $a<1$ to be chosen later. Let $\mathbf{G}$ be the random $r$-uniform hypergraph on $n$ vertices such that each hyperedge belongs to $\mathbf{G}$ independently with probability $p$. Thus, the expected number of hyperedges in $\mathbf{G}$ is $\left(\begin{array}{l}n \\ r\end{array}\right) p$, which is at least $c_{1} a n^{(g-1) /(g-2)}$. Let $2 \leq j<g$. The union of the hyperedges of any minimal cycle of length $j$ contains at most $r j-j$ vertices. The number of cycles of length $j$ whose union is a given 
set of size $i$ is at most $\left(\begin{array}{l}i \\ r\end{array}\right)^{j}$, and each such cycle has probability $p^{j}$ to belong to $\mathbf{G}$. Also, the number of hyperedges that belong to some minimal cycle of length $j$ is at most $j$ times the number of such minimal cycles. Thus, the expected number of hyperedges that belong to some minimal cycle of length $j$ is at most $c_{2} \sum_{i=r}^{r j-j}\left(\begin{array}{c}n \\ i\end{array}\right) p^{j} \leq c_{3} n^{(r-1) j} p^{j}=c_{3} a^{j} n^{j /(g-2)}$. Hence the expected number of hyperedges that belong to some cycle of length less than $g$ is at most $\sum_{j=2}^{g-1} c_{3} a^{j} n^{j /(g-2)} \leq c_{4} a^{2} n^{(g-1) /(g-2)}$. Now for $a$ sufficiently small, $C:=c_{1} a-c_{4} a^{2}>0$. That is, for $a$ sufficiently small, the expected number of hyperedges in $\mathbf{G}$ that do not belong to any cycle of length less than $g$ is more than $C n^{(g-1) /(g-2)}$. Therefore, there is some hypergraph with more than $C n^{(g-1) /(g-2)}$ hyperedges that do not belong to any cycle of length less than $g$. Take such a hypergraph and remove all hyperedges in cycles of length less than $g$.

We remark that the hypergraph may be constructed to be connected at the price of allowing the number of vertices to be in the interval $[n, n+r-2]$. To see this, if the result above is disconnected and has at least $r$ connected components, then we may add a hyperedge to reduce the number of components without creating any new cycles. If, on the other hand, the number $p$ of connected components is between 2 and $r-1$, then we may add a hyperedge containing $r-p$ new vertices to make it connected without creating any new cycles.

\section{$5 \quad$ Forbidden Subgraphs}

Given the edge set $E$ of a graph $K$, identify subsets $A \subseteq E$ with their indicator functions $\mathbf{1}_{A} \in\left(\mathbb{Z}_{2}\right)^{E}$, so that $A_{1} \triangle A_{2}$ is identified with $\mathbf{1}_{A_{1}}+\mathbf{1}_{A_{2}}$. We say that a simple cycle $C$ is generated by simple cycles $C_{1}, \ldots, C_{j}$ if $C$ is the sum (in the previous sense) of the $C_{i}$ $(1 \leq i \leq j)$, where we regard a simple cycle as its set of edges. Given an integer $g \geq 3$, say that a graph $K$ is $g$-small if $K$ is connected, has no cutpoints, and all simple cycles in $K$ are generated by simple cycles in $K$ of length $<g$. For example, if $K$ is connected, has no cutpoints, and has size $<g$, then $K$ is $g$-small. For another example, note that the usual Cayley graph of $\mathbb{Z}^{2}$, i.e., the infinite square lattice graph, is 5 -small.

Given a class $\mathcal{H}$ of graphs, write $\operatorname{Forb}(\mathcal{H})$ for the class of finite graphs that have no induced subgraph in $\mathcal{H}$. Note that if $\mathcal{H}$ is finite and consists of connected finite graphs without cutpoints, then $\mathcal{H}$ contains only $g$-small graphs for some fixed $g$. Also, if $\mathcal{H}$ is hereditary and each graph $K$ in $\mathcal{H}$ has the property that all simple cycles in $K$ are generated by simple cycles in $K$ of length $<g$, then $\operatorname{Forb}(\mathcal{H})=\operatorname{Forb}\left(\mathcal{H}^{\prime}\right)$ for some class $\mathcal{H}^{\prime}$ that contains only $g$-small graphs. Indeed, we may let $\mathcal{H}^{\prime}$ be the class of graphs in $\mathcal{H}$ that are connected and have no cutpoints.

Theorem 5.1 Let $g \geq 3$ be an integer and $\mathcal{H}$ be a collection of $g$-small graphs. The uniform ordering is the unique consistent random ordering on the class Forb $(\mathcal{H})$.

The quantitative version of this theorem follows. In it, we speak of a restricted class of (induced) embeddings of a graph $H$ in a graph $G$. We use the superscript res to denote 
the restriction in counting embeddings and in counting ordered embeddings. The restriction depends on both $H$ and $G$ and can be arbitrary, but it does not depend on orderings of $H$ and $G$. We denote by $N_{\text {ind }}^{\text {res }}(H, G)$ the number of restricted embeddings, while for any fixed orderings $<_{H},<_{G}$ of $H, G$, resp., we denote by $N_{\text {ord }}^{\text {res }}(H, G)$ the number of restricted embeddings that preserve $<_{H},<_{G}$. The proof that Theorem 5.2 implies Theorem 5.1 is the same as that of Lemma 2.1.

Theorem 5.2 Let $k, g \geq 3$ be integers and $\mathcal{H}$ be a collection of $g$-small graphs. There exists a constant $C(k, g)$ with the following property. For every graph $H \in F$ orb $(\mathcal{H})$ on $k$ vertices and $n \geq k$, there exists a graph $G \in F \operatorname{orb}(\mathcal{H})$ on $n$ vertices such that there is a restricted class of embeddings for which $N_{\text {ind }}^{\text {res }}(H, G)>0$ and for every pair of orderings $<_{G}$ of $G$ and $<_{H}$ of $H$,

$$
\left|\frac{N_{\text {ord }}^{\mathrm{res}}(H, G)}{N_{\text {ind }}^{\mathrm{res}}(H, G)}-\frac{1}{k !}\right|<\sqrt{\frac{\log n}{C(k, g) n^{1 /(g-2)}}} .
$$

Proof. Let $L$ be the number of automorphisms of $H$. Let $C(k, g)$ be the constant of Lemma 4.1. Write $m:=\left\lceil C(k, g) n^{(g-1) /(g-2)}\right\rceil$. Let $G_{0}$ be a $k$-uniform hypergraph on $n$ vertices $V$ having $m$ hyperedges $e_{1}, \ldots, e_{m}$ and girth at least $g$. Such a $G_{0}$ exists by Lemma 4.1 when $n \geq k$. Since the girth of $G_{0}$ is larger than 2, no two hyperedges share more than one vertex of $G_{0}$. Let $\mathbf{G}$ be the random graph obtained from $G_{0}$ as follows. For $1 \leq i \leq m$, let $\mathbf{H}_{i}$ be the random graph isomorphic to $H$ with vertex set $e_{i}$ induced by a uniform random bijection between $e_{i}$ and $V(H)$. Choose $\mathbf{H}_{i}$ independently. The union of all $\mathbf{H}_{i}$ is $\mathbf{G}$.

We claim that $\mathbf{G} \in \operatorname{Forb}(\mathcal{H})$. Suppose not and that $\mathbf{G}$ has an induced subgraph $K \in \mathcal{H}$. Since $H \in F \operatorname{orb}(\mathcal{H})$, it follows that $K$ is not contained entirely within any $\mathbf{H}_{i}$. Since $K$ is connected and has no cutpoints, it also follows that there is a simple cycle $C$ of $K$ that has length at least $g$ and that there is a cycle $C^{\prime}=\left\langle x_{1}, e_{r_{1}}, x_{2}, e_{r_{2}}, \ldots, x_{L}, e_{r_{L}}, x_{L+1}\right\rangle$ in $G_{0}$ and paths $P_{i} \subseteq \mathbf{H}_{r_{i}}$ joining $x_{i}$ to $x_{i+1}$ so that $C$ is the concatenation of the paths $P_{1}, \ldots, P_{L}$. Choose such a pair of cycles $C, C^{\prime}$ with $L$ a minimum. Then all $x_{i}$ are distinct. Now $C$ is generated by simple cycles in $K$ of length $<g$, each of which, being simple, lies within some $\mathbf{H}_{i}$. However, the sum of cycles is an even graph, i.e., all its vertices have even degree, whereas the intersection of $C$ with each $\mathbf{H}_{i}$ is a union of paths with distinct endpoints and thus is not an even graph. Since this intersection must be generated by the cycles that lie within $\mathbf{H}_{i}$, we obtain a contradiction, which establishes our claim.

We shall restrict to the embeddings of $H$ in $\mathbf{G}$ that embed $H$ in some hyperedge of $G_{0}$. Thus, $N_{\text {ind }}^{\text {res }}(H, \mathbf{G})=L m$.

Fix $<_{V}$ and $<_{H}$. Let $G$ be a possible value of G. Let $f(G):=N_{\text {ord }}^{\text {res }}(H, G) /(L m)$. Now $N_{\text {ord }}^{\text {res }}(H, \mathbf{G})$ has a binomial distribution with parameters $(m, L / k !)$. Thus, Chernoff's inequality yields

$$
\mathbf{P}[|f(\mathbf{G})-1 / k !| \geq D] \leq 2 \exp \left\{-2 L^{2} D^{2} m\right\}
$$

Choose

$$
D:=\sqrt{\frac{n \log n}{m}} \leq \sqrt{\frac{\log n}{C(k, g) n^{1 /(g-2)}}} .
$$


This gives

$$
\mathbf{P}[|f(\mathbf{G})-1 / k !| \geq D] \leq 2 \exp \left\{-2 L^{2} n \log n\right\} .
$$

Since this holds for every $<_{V}$ and every $<_{H}$ and the number of pairs of orderings of $V\left(G_{0}\right)$ and $V(H)$ is $n ! k !$, we obtain the result.

A similar proof clearly works for the Fraïssé class of $\mathcal{A}$-free hypergraphs of a given type and also for the Fraïssé class of abstract simplicial complexes of bounded dimension. So we have:

Theorem 5.3 The uniform ordering is the unique consistent random ordering on the class of finite $\mathcal{A}$-free hypergraphs of a given type and for the class of finite simplicial complexes of bounded dimension.

Remark 5.4 The method used in this section to prove uniqueness of consistent random orderings works also for the classes considered in Section 2.

\section{Metric Spaces}

Since there are many interesting classes of metric spaces, they provide a fertile ground for investigation. First we prove that when (essentially) no restriction is placed on the class of metric spaces, the only consistent random ordering is the uniform one. In the next section, we show that some particular classes have other consistent orderings.

Theorem 6.1 Let $S$ be an additive subsemigroup of $\mathbb{R}^{+}$. The uniform ordering is the unique consistent random ordering on the class of metric spaces with non-zero distances in $S$.

A quantitative version follows.

Theorem 6.2 Let $k, \alpha \geq 3$ be integers. There exists a constant $C^{\prime}(k, \alpha)$ with the following property. For every metric space $\langle X, d\rangle$ on $k$ vertices satisfying $d\left(x_{1}, x_{2}\right) \leq \alpha d\left(x_{3}, x_{4}\right)$ for all $x_{i} \in X$ with $x_{3} \neq x_{4}$, and for every $n \geq k$, there exists a metric space $Y$ on $n$ vertices with (non-zero) distances in the additive semigroup generated by the (non-zero) distances in $X$ and such that there is a restricted class of embeddings for which $N_{\text {ind }}^{\text {res }}(X, Y)>0$ and for every pair of orderings $<_{X}$ of $X$ and $<_{Y}$ of $Y$,

$$
\left|\frac{N_{\text {ord }}^{\text {res }}(X, Y)}{N_{\text {ind }}^{\text {res }}(X, Y)}-\frac{1}{k !}\right| \leq \sqrt{\frac{\log n}{C^{\prime}(k, \alpha) n^{1 /(\alpha-1)}}} .
$$

Proof. Let $L$ be the number of isometries of $X$, so $L \leq k$ !.

Let $C^{\prime}(k, \alpha):=C(k, \alpha+1)$, where $C(r, g)$ is the constant of Lemma 4.1. Write $m:=$ $\left\lceil C^{\prime}(k, \alpha) n^{\alpha /(\alpha-1)}\right\rceil$. Let $G_{0}$ be a $k$-uniform hypergraph on $n$ vertices $V$ having $m$ hyperedges $e_{1}, \ldots, e_{m}$ and girth at least $\alpha+1$. Such a $G_{0}$ exists by Lemma 4.1 when $n \geq k$. Note in particular that no two hyperedges share more than one vertex of $G_{0}$. Let $\mathbf{Y}$ be the random 
metric space on $V$ obtained from $G_{0}$ as follows. For $1 \leq i \leq m$, let $\left(\mathbf{X}_{i}, d_{i}\right)$ be the random metric space isometric to $X$ on $e_{i}$ induced by a uniform random bijection between $e_{i}$ and $X$. Extend the resulting metric $d$ to all pairs $z, w \in V$ by using the induced shortest-path metric for those pairs that are joined by a path of points whose consecutive distances have already been defined. Let $\beta$ be the maximum distance thereby obtained and define $d(z, w)$ to be $\beta$ if there is no path of $G_{0}$ that joins $z$ and $w$. This defines $\mathbf{Y}$. Note that the restriction of $d$ to each $\mathbf{X}_{i}$ agrees with $d_{i}$ since if $P$ is a shortest path between two points $x, y \in \mathbf{X}_{i}$, then either $P$ is contained within $\mathbf{X}_{i}$, in which case the result follows from the triangle inequality for $d_{i}$, or $P$ has length at least $\alpha$, in which case the length of $P$ is at least $\alpha d_{i}(x, y) / \alpha=d_{i}(x, y)$ by the hypothesized inequality involving $\alpha$.

We restrict to the embeddings of $X$ in $\mathbf{Y}$ that embed $X$ in some hyperedge. Thus, $N_{\text {ind }}^{\text {res }}(X, \mathbf{Y})=L m$.

Fix $<_{Y}$ and $<_{X}$. Let $Y$ be a possible value of $\mathbf{Y}$. Let $f(Y):=N_{\text {ord }}^{\text {res }}(X, Y) /(L m)$. Now $N_{\text {ord }}^{\text {res }}(X, \mathbf{Y})$ has a binomial distribution with parameters $(m, L / k !)$. Thus, Chernoff's inequality yields

$$
\mathbf{P}[|f(\mathbf{Y})-1 / k !| \geq D] \leq 2 \exp \left\{-2 L^{2} D^{2} m\right\} .
$$

Choose $D:=\sqrt{n \log n / m}$. This gives

$$
\mathbf{P}[|f(\mathbf{Y})-1 / k !| \geq D] \leq 2 \exp \left\{-2 L^{2} n \log n\right\} .
$$

Since this holds for every $<_{Y}=<_{V}$ and every $<_{X}$ and the number of pairs of orderings of $V$ and $<_{X}$ is $n ! k$ !, we obtain the result.

\section{Euclidean and Other Metric Spaces}

For certain classes of metric spaces, there is a non-uniform consistent random ordering. We begin by describing an idea of Leonard Schulman (personal communication, 2010) for randomly ordering finite subsets $X$ of Euclidean space $\mathbb{R}^{n}$. Project $\mathbb{R}^{n}$ orthogonally onto a uniformly random oriented line through the origin. Order the points of $X$ corresponding to the order of their projections on the line. Write $\mu_{X}$ for the law of this random order. By considering translations, rotations, and reflections separately, it is not hard to see that if $\phi: \mathbb{R}^{n} \rightarrow \mathbb{R}^{n}$ is an isometry, then $\mu_{\phi[X]}=\phi_{*}\left(\mu_{X}\right)$; furthermore, $\mu_{Y}$ is induced by restriction of $\mu_{X}$ when $Y \subseteq X$. In that sense, $X \mapsto \mu_{X}$ is consistent for the class of finite subsets of $\mathbb{R}^{n}$. In addition, it is consistent in the following sense: if $X \subset \mathbb{R}^{m} \subset \mathbb{R}^{n}$ for some $m<n$, then the probability measure on orderings of $X$ induced by random projections of $\mathbb{R}^{m}$ is the same as the one induced from $\mathbb{R}^{n}$. Finally, it is consistent in that whenever $\phi: X \rightarrow Y$ is an isometry of finite subsets of $\mathbb{R}^{n}$ with their induced metrics, then $\mu_{Y}=\phi_{*}\left(\mu_{X}\right)$ since $\phi$ extends to an isometry of all of $\mathbb{R}^{n}$. (That is, Euclidean space is ultrahomogeneous.)

To extend this idea, call a metric space Euclidean if it is isometric to a subset of some Euclidean space. 
Theorem 7.1 Fix an injection $f:[0, \infty) \rightarrow[0, \infty)$ with $f(0)=0$. There is a non-uniform consistent random ordering on the class of those finite metric spaces $\langle X, d\rangle$ for which $\langle X, f \circ d\rangle$ is Euclidean.

Proof. Given an isometric embedding $\phi:\langle X, f \circ d\rangle \rightarrow \mathbb{R}^{|X|}$, define the ordering $\bar{\mu}_{X}:=\phi^{*} \mu_{\phi[X]}$ pulled back from the ordering on the image of $X$ defined above. By ultrahomogeneity, $\bar{\mu}_{X}$ is independent of choice of $\phi$. However, $\bar{\mu}_{X}$ is not uniform whenever not all non-0 distances in $X$ are the same.

Examples include metric spaces of negative type, which can be defined as those metric spaces $\langle X, d\rangle$ such that $\langle X, f \circ d\rangle$ is Euclidean for $f(s):=\sqrt{s}$. In fact, it then turns out that one may also take $f(s):=s^{\alpha}$ for any $\alpha \in(0,1 / 2]$; see [Sch]. Examples of metric spaces of negative type include ultrametric spaces, spheres, hyperbolic spaces, and all $L^{p}$ spaces for $1 \leq p \leq 2$; see [Mec, Theorem 3.6] and the references there.

It should be pointed out that the Ramsey properties of classes of Euclidean metric spaces are far from being understood and it is conceivable that the above have some relevance in this context.

One can also establish Theorem 3.2, i.e., that there is a non-uniform consistent random ordering on graphs of bounded degree, by this random projection method. In order to choose distances on the vertices of a graph $G=\langle V, E\rangle$ with maximum degree at most $D$ that give a metric of negative type, fix $a<1$ such that $a /(1-a)=D$ and for $x, y \in V$, define

$$
d(x, y):= \begin{cases}0 & \text { if } x=y, \\ 1 & \text { if }\{x, y\} \in E, \\ a & \text { otherwise. }\end{cases}
$$

We use the fact [Sch] that a finite metric space has negative type iff its matrix of distances is conditionally negative semidefinite (CNSD). Here, a matrix $M$ is called CNSD if $(M v, v) \leq 0$ for all vectors $v$ that are orthogonal to the constant vectors. Let $A$ be the adjacency matrix and $J$ be the all-ones matrix indexed by the vertices. Then the distance matrix is $A+a(J-A-I)=(1-a) A-a I+a J$. When this matrix acts on a vector orthogonal to the constants, $J$ sends the vector to 0 , so the distance matrix is CNSD iff $(1-a) A-a I$ is CNSD iff $-(D I-A)$ is CNSD. But in fact, this matrix is negative semidefinite (NSD) as seen, e.g., by comparison to the graph Laplacian matrix. Here, a matrix $M$ is called NSD if $(M v, v) \leq 0$ for all vectors $v$.

It seems that using random distances, one can also use this method for uniform hypergraphs, but this appears even harder than the method used in the discussion of Remark 3.5.

If one wishes, one can define the random ordering on finite subsets of Euclidean spaces in all dimensions at once by considering finite subsets $X$ of Hilbert space $\mathscr{H}:=\ell^{2}(\mathbb{N})$ instead. For that case, let $\left\langle v_{n} ; n \geq 0\right\rangle$ be any orthonormal basis of $\mathscr{H}$ and let $\left\langle Z_{n} ; n \geq 0\right\rangle$ be independent standard normal random variables. Order $X$ by the order on the real numbers $\sum_{n} Z_{n}\left\langle x, v_{n}\right\rangle$ for $x \in X$; this sum converges a.s. by Kolmogorov's Three-Series Theorem because $\sum_{n}\left|\left\langle x, v_{n}\right\rangle\right|^{2}<\infty$. The sum has a normal distribution with variance $\|x\|^{2}$. The 
spherical symmetry of the standard multivariate normal distribution in Euclidean spaces shows that this random ordering does not depend on the choice of basis and agrees with the random ordering $\mu_{X}$ previously defined.

This is related to the Lévy-Ciesielski construction of Brownian motion: First, note that $\mathbb{R}^{+}$has negative type, as we can see by embedding $\mathbb{R}^{+}$into $L^{2}\left(\mathbb{R}^{+}\right)$via mapping $x \in \mathbb{R}^{+}$to the function $s \mapsto \mathbf{1}_{[0, x]}(s)$. Second, identify $L^{2}\left(\mathbb{R}^{+}\right)$with $\mathscr{H}$ by taking as orthonormal basis the Haar basis. Then it is not hard to see that $\sum_{n} Z_{n}\left\langle\mathbf{1}_{[0, x]}, v_{n}\right\rangle$ a.s. converges uniformly for $x$ belonging to any compact set. Since it is a mean-0 Gaussian process with covariance $(x, y) \mapsto\left\langle\mathbf{1}_{[0, x]}, \mathbf{1}_{[0, y]}\right\rangle=\min \{x, y\}$, it is standard Brownian motion. This is precisely the Lévy-Ciesielski construction.

This concludes the discussion of consistent random orderings on specific classes of finite structures. The rest of the paper will connect this with the unique ergodicity phenomena.

\section{Topological Dynamics and Unique Ergodicity}

We prove here some facts concerning unique ergodicity for amenable groups that will be used in subsequent sections. First we note the following general fact.

Proposition 8.1 Let $\Gamma$ be a topological group. Then $\Gamma$ is amenable iff the universal minimal flow of $\Gamma$ admits an invariant measure. Moreover, $\Gamma$ is uniquely ergodic iff the universal minimal flow of $\Gamma$ is uniquely ergodic.

Proof. The first statement is obvious, since every flow contains a minimal subflow. For the second, it is enough to show that if $X$ is a uniquely ergodic $\Gamma$-flow, $Y$ is a $\Gamma$-flow and $\pi: X \rightarrow Y$ is a surjective, continuous $\Gamma$-map, then $Y$ is uniquely ergodic. For that it is enough again to show that if $\nu$ is a $\Gamma$-invariant measure on $Y$, then there is a $\Gamma$-invariant measure $\hat{\nu}$ such that $\pi_{*} \hat{\nu}=\nu$.

First note that there is a measure $\mu_{0}$ on $X$ such that $\pi_{*} \mu_{0}=\nu$. Indeed the set

$$
\left\{\pi_{*} \mu ; \mu \text { is a measure on } X\right\}
$$

is a compact, convex set, containing all Dirac measures, thus, by Hahn-Banach, it contains all measures on $Y$. It follows that the set of all measures $\mu$ on $X$ with $\pi_{*} \mu=\nu$ is a non-empty, compact, convex set of measures on which $\Gamma$ acts continuously by affine transformations (the action given as usual by: $\int f d(g \cdot \mu)=\int\left(g^{-1} \cdot f\right) d \mu$, for $g \in \Gamma, f \in C(X)$, where $\left.g \cdot f(x)=f\left(g^{-1} \cdot x\right)\right)$. Since $\Gamma$ is amenable, this action has a fixed point $\hat{\nu}$ (see [BHV, G.1.7]) and thus $\hat{\nu}$ is as required.

Next we provide a characterization of unique ergodicity in the case of compactly approximable groups.

Let $\Gamma$ be a compactly approximable topological group and let $\Delta_{0} \subseteq \Delta_{1} \subseteq \cdots$ be a sequence of compact subgroups with $\overline{\bigcup_{n} \Delta_{n}}=\Gamma$. Let $\mu_{n}$ be the Haar measure of $\Delta_{n}$. Let $X$ 
be a $\Gamma$-flow. For $f \in C(X), n \in \mathbb{N}$, define the averaging operator $A_{n}$ on $C(X)$ by

$$
A_{n}(f)(x):=\int_{\Delta_{n}} f\left(g^{-1} \cdot x\right) d \mu_{n}(g) .
$$

The following is an analog of 4.9 in Glasner [Gl].

Theorem 8.2 In the preceding notation, the following are equivalent:

(i) $\forall f \in C(X) \exists f^{*} \in \mathbb{C}\left(A_{n}(f)\right.$ converges uniformly to $\left.f^{*}\right)$,

(ii) $\forall f \in C(X) \exists f^{*} \in \mathbb{C}\left(A_{n}(f)\right.$ converges pointwise to $\left.f^{*}\right)$,

(iii) There is a $\Gamma$-invariant measure $\mu$ on $X$ such that

$$
\forall f \in C(X)\left(A_{n}(f) \text { converges pointwise to } \int f d \mu\right)
$$

(iv) The $\Gamma$-flow $X$ is uniquely ergodic.

Proof. (i) $\Rightarrow$ (ii) is obvious.

(ii) $\Rightarrow$ (iii): Put $A(f):=\lim _{n \rightarrow \infty} A_{n}(f)(x) \in \mathbb{C}$ (for any $x \in X$ ). Then $A$ is a positive linear functional on $C(X)$ with $A(1)=1$, so by the Riesz Representation Theorem there is a measure $\mu$ on $X$ with

$$
A(f)=\int f d \mu .
$$

We shall show that $\mu$ is $\Gamma$-invariant. It is of course enough to show that it is $\left(\bigcup_{n} \Delta_{n}\right)$-invariant or equivalently that $A$ is $\left(\bigcup_{n} \Delta_{n}\right)$-invariant, where $\Gamma$ acts on $C(X)$ by $g \cdot f(x):=f\left(g^{-1} \cdot x\right)$. Fix $h \in \bigcup_{n} \Delta_{n}$. Then

$$
\begin{aligned}
A(h \cdot f)(x) & =\lim _{n \rightarrow \infty} \int_{\Delta_{n}}(h \cdot f)\left(g^{-1} \cdot x\right) d \mu_{n}(g) \\
& =\lim _{n \rightarrow \infty} \int_{\Delta_{n}} f\left(h^{-1} g^{-1} \cdot x\right) d \mu_{n}(g) \\
& =\lim _{n \rightarrow \infty} \int_{\Delta_{n}} f\left((g h)^{-1} \cdot x\right) d \mu_{n}(g) \\
& =\lim _{n \rightarrow \infty} \int_{\Delta_{n}} f\left(g^{-1} \cdot x\right) d \mu_{n}(g) \\
& =A(f)(x)
\end{aligned}
$$

by the invariance of Haar measure.

(iii) $\Rightarrow$ (iv): Let $\mu$ be a $\Gamma$-invariant measure with $A_{n}(f)(x) \rightarrow \int f d \mu=f^{*}$ pointwise. Let $\nu$ be any $\Gamma$-invariant measure. We shall show that $\mu=\nu$. By Lebesgue Dominated Convergence, we have

$$
\int A_{n}(f)(x) d \nu(x) \rightarrow \int f^{*} d \nu=f^{*}=\int f d \mu .
$$


But also by Fubini and the $\Gamma$-invariance of $\nu$,

$$
\begin{aligned}
\int A_{n}(f)(x) d \nu(x) & =\int\left(\int_{\Delta_{n}} f\left(g^{-1} \cdot x\right) d \mu_{n}(g)\right) d \nu(x) \\
& =\int_{\Delta_{n}}\left(\int f\left(g^{-1} \cdot x\right) d \nu(x)\right) d \mu_{n}(g) \\
& =\int_{\Delta_{n}}\left(\int f d \nu\right) d \mu_{n}(g)=\int f d \nu
\end{aligned}
$$

i.e., $\int f d \mu=\int f d \nu, \forall f \in C(X)$, so $\mu=\nu$.

(iv) $\Rightarrow$ (i): Let $\mu$ be the unique $\Gamma$-invariant measure. If (i) fails, there is $f \in C(X)$ such that $A_{n}(f)$ does not converge uniformly to $\int f d \mu$. Fix then $\epsilon>0$ such that for infinitely many $n$, there is $x_{n} \in X$ with $\left|A_{n}(f)\left(x_{n}\right)-\int f d \mu\right| \geq \epsilon$. For such $n$, let $\rho_{n}$ be the measure on $X$ defined by

$$
\int h d \rho_{n}:=\int_{\Delta_{n}} h\left(g^{-1} \cdot x_{n}\right) d \mu_{n}(g)
$$

for $h \in C(X)$. Thus

So

$$
\int f d \rho_{n}=A_{n}(f)\left(x_{n}\right)
$$

$$
\left|\int f d \rho_{n}-\int f d \mu\right| \geq \epsilon
$$

By compactness there is a measure $\rho_{\infty}$ and a subsequence $\left(\rho_{n_{i}}\right)$ converging to $\rho_{\infty}$ in the weak*-topology of measures. Thus

$$
\left|\int f d \rho_{\infty}-\int f d \mu\right| \geq \epsilon
$$

so $\rho_{\infty} \neq \mu$. We shall show that $\rho_{\infty}$ is $\Gamma$-invariant, which is a contradiction. Fix $g_{0} \in \bigcup_{n} \Delta_{n}$. Then for any $h \in C(X)$,

$$
\begin{aligned}
\int\left(g_{0} \cdot h\right) d \rho_{\infty} & =\lim _{i \rightarrow \infty} \int\left(g_{0} \cdot h\right) d \rho_{n_{i}} \\
& =\lim _{i \rightarrow \infty}\left[\int_{\Delta_{n_{i}}}\left(g_{0} \cdot h\right)\left(g^{-1} \cdot x_{n_{i}}\right) d \mu_{n_{i}}(g)\right] \\
& =\lim _{i \rightarrow \infty}\left[\int_{\Delta_{n_{i}}} h\left(\left(g g_{0}\right)^{-1} \cdot x_{n_{i}}\right) d \mu_{n_{i}}(g)\right] \\
& =\lim _{i \rightarrow \infty} \int_{\Delta_{n_{i}}} h\left(g^{-1} \cdot x_{n_{i}}\right) d \mu_{n_{i}}(g) \\
& =\lim _{i \rightarrow \infty} \int h d \rho_{n_{i}} \\
& =\int h d \rho_{\infty} .
\end{aligned}
$$




\section{Universal Minimal Flow of Automorphism Groups and Unique Ergodicity}

Throughout the rest of this paper we shall work in the following context developed in [KPT] (although our notation will be slightly different).

Consider a Fraïssé class $\mathcal{K}$ in a language $L$. Let $L^{*}:=L \cup\{<\}$ be the language obtained by adding a binary relation symbol $<$ to $L$. A structure $\boldsymbol{A}^{*}$ for $L^{*}$ has the form $\boldsymbol{A}^{*}=\left\langle\boldsymbol{A},<_{\boldsymbol{A}}\right\rangle$, where $\boldsymbol{A}$ is a structure for $L$ and $<_{\boldsymbol{A}}$ is a binary relation on $A$ ( $=$ the universe of $\boldsymbol{A}$ ). We often write more simply $\langle\boldsymbol{A},<\rangle$ for $\left\langle\boldsymbol{A},<_{\boldsymbol{A}}\right\rangle$. A class $\mathcal{K}^{*}$ of finite structures in $L^{*}$ is called an order class if $\left(\langle\boldsymbol{A},<\rangle \in \mathcal{K}^{*} \Rightarrow<\right.$ is a linear ordering on $\left.A\right)$. For such $\boldsymbol{A}^{*}=\langle\boldsymbol{A},<\rangle$, let $\boldsymbol{A}^{*}\lceil L:=\boldsymbol{A}$.

We say that an order class $\mathcal{K}^{*}$ on $L^{*}$, closed under isomorphism, is an order expansion of $\mathcal{K}$ if $\mathcal{K}=\mathcal{K}^{*} \mid L:=\left\{\boldsymbol{A}^{*}\left\lceil L ; \boldsymbol{A}^{*} \in \mathcal{K}^{*}\right\}\right.$. In this case, if $\boldsymbol{A} \in \mathcal{K}$ and $\boldsymbol{A}^{*}:=\langle\boldsymbol{A},<\rangle \in \mathcal{K}^{*}$, we say that $<$ is a $\mathcal{K}^{*}$-admissible ordering of $\boldsymbol{A}$. The order expansion $\mathcal{K}^{*}$ of $\mathcal{K}$ is reasonable if for every $\boldsymbol{A}, \boldsymbol{B} \in \mathcal{K}$ with $\boldsymbol{A} \subseteq \boldsymbol{B}$ and any $\mathcal{K}^{*}$-admissible ordering $<_{\boldsymbol{A}}$ on $\boldsymbol{A}$, there is a $\mathcal{K}^{*}$-admissible ordering $<_{\boldsymbol{B}}$ on $\boldsymbol{B}$ such that $<_{\boldsymbol{A}} \subseteq<_{\boldsymbol{B}}$.

If $\mathcal{K}$ is a Fraïssé class with $\boldsymbol{K}=\operatorname{Flim}(\mathcal{K})$ and $\mathcal{K}^{*}$ is a reasonable order expansion of $\mathcal{K}$, we denote by $X_{\mathcal{K}^{*}}$ the space of linear orderings $<$ on $K$ such that for any finite substructure $\boldsymbol{A}$ of $\boldsymbol{K},<\uparrow A$ is $\mathcal{K}^{*}$-admissible on $\boldsymbol{A}$. We call these the $\mathcal{K}^{*}$-admissible orderings on $\boldsymbol{K}$. They form a compact, non-empty subspace of $2^{K^{2}}$, with the product topology, on which the group $\Gamma:=\operatorname{Aut}(\boldsymbol{K})$ acts continuously. Thus $X_{\mathcal{K}^{*}}$ is a $\Gamma$-flow.

If $\mathcal{K}^{*}$ is an order expansion of $\mathcal{K}$, we say that $\mathcal{K}^{*}$ satisfies the ordering property (OP) if for every $\boldsymbol{A} \in \mathcal{K}$, there is $\boldsymbol{B} \in \mathcal{K}$ such that for every pair of $\mathcal{K}^{*}$-admissible orderings $<_{\boldsymbol{A}}$ on $\boldsymbol{A}$ and $<_{\boldsymbol{B}}$ on $\boldsymbol{B},\left\langle\boldsymbol{A},<_{\boldsymbol{A}}\right\rangle$ can be embedded in $\left\langle\boldsymbol{B},<_{\boldsymbol{B}}\right\rangle$.

We also say that a class $\mathcal{K}$ of finite structures in a given language $L$ has the $\boldsymbol{R a m s e y}$ Property $(\boldsymbol{R P})$ if for any $\boldsymbol{A}, \boldsymbol{B} \in \mathcal{K}$ with $\boldsymbol{A} \leq \boldsymbol{B}$ (i.e., $\boldsymbol{A}$ can be embedded in $\boldsymbol{B}$ ), there is $\boldsymbol{C} \in \mathcal{K}$ with $\boldsymbol{B} \leq \boldsymbol{C}$ such that for any coloring $c:\left(\begin{array}{l}\boldsymbol{C} \\ \boldsymbol{A}\end{array}\right) \rightarrow\{1, \ldots, k\}$, there is $\boldsymbol{B}^{\prime} \in\left(\begin{array}{l}\boldsymbol{C} \\ \boldsymbol{B}\end{array}\right)$ such that $c \uparrow\left(\begin{array}{l}\boldsymbol{B}^{\prime} \\ \boldsymbol{A}\end{array}\right)$ is constant. Here for $\boldsymbol{D} \leq \boldsymbol{E}$, we let $\left(\begin{array}{c}\boldsymbol{E} \\ \boldsymbol{D}\end{array}\right):=$ the set of all substructures of $\boldsymbol{E}$ isomorphic to $\boldsymbol{D}$.

The following is shown in [KPT, 7.4, 10.8].

Theorem 9.1 [KPT] Let $\mathcal{K}$ be a Fraïssé class in $L$ and $\mathcal{K}^{*}$ a reasonable order expansion of $\mathcal{K}$ in $L^{*}$ that is also a Fraïssé class. Let $\boldsymbol{K}:=\operatorname{Flim}(\mathcal{K})$ and $\Gamma:=\operatorname{Aut}(\boldsymbol{K})$. Then the following are equivalent:

(i) $X_{\mathcal{K}^{*}}$ is a minimal $\Gamma$-flow;

(ii) $\mathcal{K}^{*}$ satisfies the OP.

Moreover, the following are equivalent:

(a) $X_{\mathcal{K}^{*}}$ is the universal minimal flow of $\Gamma$; 


\section{(b) $\mathcal{K}^{*}$ satisfies the $\mathrm{OP}$ and the RP.}

We call $\left(\mathcal{K}, \mathcal{K}^{*}\right)$ an excellent pair if $\mathcal{K}$ is a Fraïssé class in $L, \mathcal{K}^{*}$ is a Fraïssé, reasonable order expansion of $\mathcal{K}$ in $L^{*}$, and $\mathcal{K}^{*}$ satisfies the OP and the RP. It was shown in [KPT, 9.2] that if $\mathcal{K}$ admits an excellent pair $\left(\mathcal{K}, \mathcal{K}^{*}\right)$, then $\mathcal{K}^{*}$ is essentially unique. We then call $\mathcal{K}^{*}$ a companion of $\mathcal{K}$.

The paper $[\mathrm{KPT}]$ contains many examples of excellent pairs $\left(\mathcal{K}, \mathcal{K}^{*}\right)$ including, e.g., $\mathcal{K}=$ $\mathcal{A}$-free hypergraphs of a given type, metric spaces with distances in a given countable additive subsemigroup of $\mathbb{R}^{+}$(see also [N1] here), vector spaces over a given finite field, etc., with corresponding companions $\mathcal{K}^{*}=$ ordered graphs, ordered $\mathcal{A}$-free hypergraphs of a given type, ordered metric spaces with distances in a given countable additive subsemigroup of $\mathbb{R}^{+}$, lexicographically ordered vector spaces over a given finite field (i.e., with an ordering induced lexicographically by an arbitrary ordering of a basis), etc. There are also examples of $\mathcal{K}$ that have no companion (see $[\mathrm{J}],[\mathrm{N} 2],[\mathrm{KS}]$ ), including the class of finite distributive lattices (see $[\mathrm{KS}]$ ).

If $\mathcal{K}$ is a Fraïssé class with $\boldsymbol{K}=\operatorname{Flim}(\mathcal{K})$ and $\mathcal{K}^{*}$ is a reasonable order expansion of $\mathcal{K}$, the compact space $X_{\mathcal{K}^{*}}$ is 0 -dimensional, i.e., has a basis consisting of clopen sets. For each finite substructure $\boldsymbol{A} \subseteq \boldsymbol{K}$ and each $\mathcal{K}^{*}$-admissible ordering $<$ on $\boldsymbol{A}$, let

$$
N_{\boldsymbol{A},<}:=\left\{\prec \in X_{\mathcal{K}^{*}} ; \prec \mid A=<\right\} .
$$

This is a clopen basis in $X_{\mathcal{K}^{*}}$ and the class of the sets $N_{\boldsymbol{A},<}$ generates the Borel $\sigma$-algebra of $X_{\mathcal{K}^{*}}$.

Thus, if $\mu$ is a measure on $X_{\mathcal{K}^{*}}$, then $\mu$ is completely determined by the values $\mu\left(N_{\boldsymbol{A},<}\right)$ for $\boldsymbol{A} \subseteq \boldsymbol{K}$ and $\langle\boldsymbol{A},<\rangle \in \mathcal{K}^{*}$. These satisfy the obvious relations:

(i) For $\boldsymbol{A} \in \mathcal{K}, \boldsymbol{A} \subseteq \boldsymbol{K}$,

$$
\sum\left\{\mu\left(N_{\boldsymbol{A},<}\right) ;\langle\boldsymbol{A},<\rangle \in \mathcal{K}^{*}\right\}=1
$$

(ii) For $\boldsymbol{A}, \boldsymbol{B} \in \mathcal{K}, \boldsymbol{A} \subseteq \boldsymbol{B} \subseteq \boldsymbol{K}$,

$$
\mu\left(N_{\boldsymbol{A},<}\right)=\sum\left\{\mu\left(N_{\boldsymbol{B},<^{\prime}}\right) ;\left\langle\boldsymbol{B},<^{\prime}\right\rangle \in \mathcal{K}^{*},<\subseteq<^{\prime}\right\}
$$

Moreover any map $N_{\boldsymbol{A},<} \mapsto \mu\left(N_{\boldsymbol{A},<}\right) \in[0,1]$ that satisfies these conditions extends uniquely to a measure on $X_{\mathcal{K}^{*}}$.

In fact, if $\boldsymbol{A}_{0} \subseteq \boldsymbol{A}_{1} \subseteq \cdots$ is a sequence of finite substructures of $\boldsymbol{K}$ with $\boldsymbol{K}=\bigcup_{n} \boldsymbol{A}_{n}$, the sets $N_{\boldsymbol{A}_{n},<}$, for $\left\langle\boldsymbol{A}_{n},<\right\rangle \in \mathcal{K}^{*}$, form in an obvious way a finite branching tree (where the children of $N_{\boldsymbol{A}_{n},<}$ are all $N_{\boldsymbol{A}_{n+1},<^{\prime}}$ where $<\subseteq<^{\prime}$ ) and a measure $\mu$ on $X_{\mathcal{K}^{*}}$ is completely determined by the values $\mu\left(N_{\boldsymbol{A}_{n},<}\right)$. Conversely, any map $N_{\boldsymbol{A}_{n},<} \mapsto \mu\left(N_{\boldsymbol{A}_{n},<}\right) \in[0,1]$ such that

$$
\sum\left\{\mu\left(N_{\boldsymbol{A}_{0},<}\right) ;\left\langle\boldsymbol{A}_{0},<\right\rangle \in \mathcal{K}^{*}\right\}=1
$$

and

$$
\mu\left(N_{\boldsymbol{A}_{n},<}\right)=\sum\left\{\mu\left(N_{\boldsymbol{A}_{n+1},<^{\prime}}\right) ;\left\langle\boldsymbol{A}_{n+1},<^{\prime}\right\rangle \in \mathcal{K}^{*},<\subseteq<^{\prime}\right\}
$$


extends uniquely to a measure on $X_{\mathcal{K}^{*}}$.

Let $\mathcal{K}$ be a Fraïssé class in $L$ and $\mathcal{K}^{*}$ a reasonable order expansion of $\mathcal{K}$ in $L^{*}$. A consistent random $\mathcal{K}^{*}$-admissible ordering on $\mathcal{K}$ is a map that assigns to each $\boldsymbol{A} \in \mathcal{K}$ a probability measure $\mu_{\boldsymbol{A}}$ on the set of $\mathcal{K}^{*}$-admissible orderings on $\boldsymbol{A}$ that is isomorphism invariant (i.e., if $\pi: \boldsymbol{A} \rightarrow \boldsymbol{B}$ is an isomorphism, then $\pi_{*} \mu_{\boldsymbol{A}}=\mu_{\boldsymbol{B}}$ ) and satisfies the following for each $\boldsymbol{A} \subseteq \boldsymbol{B}$ in $\mathcal{K}$ and $\langle\boldsymbol{A},<\rangle \in \mathcal{K}^{*}$ :

$$
\mu_{\boldsymbol{A}}(<)=\sum\left\{\mu_{\boldsymbol{B}}\left(<^{\prime}\right) ;\left\langle\boldsymbol{B},<^{\prime}\right\rangle \in \mathcal{K}^{*},<\subseteq<^{\prime}\right\}
$$

where we put $\mu_{\boldsymbol{A}}(<):=\mu_{\boldsymbol{A}}(\{<\})$.

It is clear that if $\left(\mu_{\boldsymbol{A}}\right)_{\boldsymbol{A} \in \mathcal{K}}$ is a consistent random $\mathcal{K}^{*}$-admissible ordering on $\mathcal{K}$, then we can define a $\Gamma$-invariant measure $\mu$ on $X_{\mathcal{K}^{*}}$, where $\Gamma:=\operatorname{Aut}(\boldsymbol{K}), \boldsymbol{K}:=\operatorname{Flim}(\mathcal{K})$, as follows:

$$
\mu\left(N_{\boldsymbol{A},<}\right):=\mu_{\boldsymbol{A}}(<) .
$$

Conversely, given such a $\Gamma$-invariant measure $\mu$ on $X_{\mathcal{K}^{*}}$, we can define a consistent random $\mathcal{K}^{*}$-admissible ordering of $\mathcal{K}$ by

$$
\mu_{\boldsymbol{A}}(<):=\mu\left(N_{\boldsymbol{A}^{\prime},<^{\prime}}\right),
$$

where $\boldsymbol{A}^{\prime} \subseteq \boldsymbol{K}$ and $\langle\boldsymbol{A},<\rangle \cong\left\langle\boldsymbol{A}^{\prime},<^{\prime}\right\rangle$. By the ultrahomogeneity of $\boldsymbol{K}$, this is well-defined.

Thus $\Gamma$-invariant measures on $X_{\mathcal{K}^{*}}$ can be identified with consistent random $\mathcal{K}^{*}$-admissible orderings on $\mathcal{K}$.

We then have, using Proposition 8.1:

Proposition 9.2 Let $\left(\mathcal{K}, \mathcal{K}^{*}\right)$ be an excellent pair. Let $\boldsymbol{K}:=\operatorname{Flim}(\mathcal{K})$. Then $\Gamma:=\operatorname{Aut}(\boldsymbol{K})$ is amenable iff $\mathcal{K}$ admits a consistent random $\mathcal{K}^{*}$-admissible ordering. Moreover, $\Gamma$ is uniquely ergodic iff $\mathcal{K}$ admits a unique consistent random $\mathcal{K}^{*}$-admissible ordering.

If $\left(\mathcal{K}, \mathcal{K}^{*}\right)$ is an excellent pair for which every linear ordering on each given $\boldsymbol{A} \in \mathcal{K}$ is $\mathcal{K}^{*}$ admissible (in this case, we write $\mathcal{K}^{*}=\mathcal{K} * \mathcal{L O}$ ), then there is an obvious $\operatorname{Aut}(\boldsymbol{K})$-invariant measure $\mu$ on $X_{\mathcal{K}^{*}}$, which in this case is the space of all linear orderings on $K$, given by

$$
\mu\left(N_{\boldsymbol{A},<}\right):=\frac{1}{n !},
$$

where $n:=|A|$; we call this the uniform measure on $X_{\mathcal{K}^{*}}$. Thus we have the following:

Proposition 9.3 Let $\left(\mathcal{K}, \mathcal{K}^{*}\right)$ be an excellent pair such that $\mathcal{K}^{*}=\mathcal{K} * \mathcal{L O}$. Then $\operatorname{Aut}(\boldsymbol{K})$ is amenable for $\boldsymbol{K}:=\operatorname{Flim}(\mathcal{K})$.

As we mentioned earlier in this section, examples of classes $\mathcal{K}$ of finite structures for which $(\mathcal{K}, \mathcal{K} * \mathcal{L O})$ is an excellent pair include the following (see [KPT, Sections 6 and 8]): pure sets, $\mathcal{A}$-free hypergraphs of a given type, metric spaces with (non-zero) distances in a given countable additive subsemigroup of $\mathbb{R}^{+}$, etc. 


\section{Order Transitivity and Unique Ergodicity}

We shall discuss here a simple criterion for unique ergodicity and use it to provide our first examples of uniquely ergodic automorphism groups. Given an excellent pair $\left(\mathcal{K}, \mathcal{K}^{*}\right)$, a structure $\boldsymbol{A} \in \mathcal{K}$ is $\mathcal{K}^{*}$-order transitive if $\operatorname{Aut}(\boldsymbol{A})$ acts transitively on the set of $\mathcal{K}^{*}$ admissible orderings on $\boldsymbol{A}$. We now have:

Proposition 10.1 Let $\left(\mathcal{K}, \mathcal{K}^{*}\right)$ be an excellent pair, let $\boldsymbol{K}:=\operatorname{Flim}(\mathcal{K})$ and assume that $\Gamma:=\operatorname{Aut}(\boldsymbol{K})$ is amenable. If the class of order-transitive structures in $\mathcal{K}$ is cofinal, i.e., for every $\boldsymbol{A} \in \mathcal{K}$, there is an order-transitive $\boldsymbol{B} \in \mathcal{K}$ with $\boldsymbol{A} \subseteq \boldsymbol{B}$, then $\Gamma$ is uniquely ergodic.

Proof. By Theorem 9.1 and Proposition 8.1, it is enough to show that $X_{\mathcal{K}^{*}}$ is uniquely ergodic. Since $\Gamma=\operatorname{Aut}(\boldsymbol{K})$ is amenable, there is a $\Gamma$-invariant measure on $X_{\mathcal{K}^{*}}$. Fix any such measure $\mu$. By hypothesis, there is a sequence $\boldsymbol{A}_{0} \subseteq \boldsymbol{A}_{1} \subseteq \cdots$ of finite substructures of $\boldsymbol{K}$ with $\bigcup_{n} \boldsymbol{A}_{n}=\boldsymbol{K}$ and each $\boldsymbol{A}_{n}$ order transitive. It follows that for each $\mathcal{K}^{*}$-admissible order $<$ on $\boldsymbol{A}_{n}$, we have $\mu\left(N_{\boldsymbol{A}_{n},<}\right)=1 / k_{n}$, where $k_{n}$ is the cardinality of the set of $\mathcal{K}^{*}$ admissible orders on $\boldsymbol{A}_{n}$. Thus $\mu$ is uniquely determined.

Here are some examples of Hrushovski classes of finite structures satisfying the hypothesis of the previous proposition:

i) $\mathcal{K}=$ pure sets, $\mathcal{K}^{*}=$ linear orderings. Then $\boldsymbol{K}=\operatorname{Flim}(\mathcal{K})=\mathbb{N}$ and $\operatorname{Aut}(\boldsymbol{K})=S_{\infty}$. So $S_{\infty}$ is uniquely ergodic (Glasner and Weiss [GW1]).

ii) $\mathcal{K}=$ equivalence relations, $\mathcal{K}^{*}=$ equivalence relations with convex orderings, i.e., orderings in which each equivalence class is convex (see [KPT, Section 6]). Then $\boldsymbol{K}=$ the equivalence relation on $\mathbb{N}$ that has infinitely many classes, each infinite, so $\operatorname{Aut}(\boldsymbol{K}) \cong S_{\infty} \ltimes S_{\infty}^{\mathbb{N}}$ (where $S_{\infty}$ acts on $S_{\infty}^{\mathbb{N}}$ by shift) is uniquely ergodic.

iii) Let $\boldsymbol{K}:=\langle\mathbb{N}<\mathbb{N}, \emptyset, p\rangle$, where $\emptyset$ is the empty sequence and $p$ is the prefix map, $p(s):=$ $s \uparrow(n-1)$ for $s \in \mathbb{N}^{n}$ for $n>0$ and $p(\emptyset):=\emptyset$. Then $\boldsymbol{K}$ is a Fraïssé structure. Let $\mathcal{K}:=\operatorname{Age}(\boldsymbol{K})$. The elements of $\mathcal{K}$ are the structures isomorphic to finite subtrees $T$ of $\mathbb{N}<\mathbb{N}$, i.e., finite subsets $T \subseteq \mathbb{N}^{<\mathbb{N}}$ containing $\emptyset$ and having the property that if $s \in \mathbb{N}^{n}$, $n>0$, and $s \in T$, then for every $m<n$, we have $s\left\lceil m \in T\right.$. We define the class $\mathcal{K}^{*}$ by saying that $<$ is a $\mathcal{K}^{*}$-admissible ordering on $T$ as above if $s, t \in T, s\lceil m=t\lceil m$ (for $m<\operatorname{length}(s)$, length $(t))$ and $s<u<t$, imply $u\left\lceil m=s\left\lceil m\right.\right.$. Then $\left(\mathcal{K}, \mathcal{K}^{*}\right)$ is excellent and $\mathcal{K}$ is a Hrushovski class (see [KR, Section 6.11] and [N1, Chapter 2]). Moreover, if $T_{n}:=\left\{s \in \mathbb{N}^{<\mathbb{N}} ; \operatorname{length}(s) \leq n\right.$ and $\left.\forall i<\operatorname{length}(s)\left(s_{i}<n\right)\right\}$, then $\left\{T_{n} ; n \geq 0\right\}$ is a cofinal class of order-transitive structures in $\mathcal{K}$. Thus $\operatorname{Aut}(\boldsymbol{K}) \cong \operatorname{Aut}\left(T_{\infty}\right)$, where $T_{\infty}$ is the rooted $\aleph_{0}$-regular tree, is uniquely ergodic.

iv) More generally, let $S \subseteq(0, \infty)$ be countable, let $\mathcal{U}_{S}:=$ the class of finite ultrametric spaces with distances in $S$ and $\mathcal{U}_{S}^{*}:=$ the class of all convexly ordered finite ultrametric spaces, where an ordering is convex if metric balls are convex. Then $\left(\mathcal{U}_{S}, \mathcal{U}_{S}^{*}\right)$ is excellent and $\mathcal{U}_{S}$ is Hrushovski. Moreover the order-transitive $\boldsymbol{A} \in \mathcal{U}_{S}$ are cofinal 
(see [N1, Chapter 2]). Here $\boldsymbol{U}_{S}^{u l t}:=\mathrm{Flim}\left(\mathcal{U}_{S}\right)$ is the Urysohn ultrametric space with distances in $S$ and thus $\operatorname{Aut}\left(\boldsymbol{U}_{S}^{u l t}\right)=\operatorname{Iso}\left(\boldsymbol{U}_{S}^{u l t}\right)$ is uniquely ergodic. (The case $S=\left\{2^{-n} ; n \in \mathbb{N}\right\}$ corresponds to the previous example.)

v) Let $F$ be a finite field and let $\mathcal{K}_{F}$ be the class of finite-dimensional vector spaces over $F$. Fix an ordering of $F$ in which $0 \in F$ is least. Let $\mathcal{K}_{F}^{*}$ be the class of naturally ordered vector spaces, where a natural order is one induced lexicographically by an ordering of a basis. Then $\left(\mathcal{K}_{F}, \mathcal{K}_{F}^{*}\right)$ is an excellent pair and $\mathcal{K}_{F}$ is Hrushovski (see [KPT, Section 6]). Clearly every $\boldsymbol{A} \in \mathcal{K}_{F}$ is order transitive. Now $\boldsymbol{V}_{\infty, F}:=\operatorname{Flim}\left(\mathcal{K}_{F}\right)$ is the (countably) infinite-dimensional vector space over $F$ and $\operatorname{Aut}\left(\boldsymbol{V}_{\infty, F}\right)=\operatorname{GL}\left(V_{\infty, F}\right)$ is the general linear group of $\boldsymbol{V}_{\infty, F}$. Thus $\mathrm{GL}\left(\boldsymbol{V}_{\infty, F}\right)$ is uniquely ergodic.

Remark 10.2 Let $\boldsymbol{V} \subseteq \boldsymbol{V}_{\infty, F}$ be a finite-dimensional vector space over $F$. Then the number of $\mathcal{K}_{F}^{*}$-admissible orders on $\boldsymbol{V}$ is equal to $|\mathrm{GL}(\boldsymbol{V})|$ and if $\mu$ is the unique invariant measure, then $\mu\left(N_{\boldsymbol{V},<}\right)=\frac{1}{|G L(\boldsymbol{V})|}$. Thus if $\boldsymbol{V} \subseteq \boldsymbol{W} \subseteq \boldsymbol{V}_{\infty, F}$ and $<$ is a $\mathcal{K}_{F}^{*}$-admissible order on $\boldsymbol{V}$, then the number of $\mathcal{K}_{F}^{*}$-admissible orders on $\boldsymbol{W}$ that extend $<$ is equal to $\frac{|\mathrm{GL}(\boldsymbol{W})|}{|\mathrm{GL}(\boldsymbol{V})|}$.

\section{A Quantitative Ordering Property and Unique Er- godicity}

We formulate here a quantitative version of the ordering property and show that it implies unique ergodicity for automorphism groups.

Let $\mathcal{K}$ be a Fraïssé class and $\mathcal{K}^{*}$ be an order expansion of $\mathcal{K}$. We say that $\mathcal{K}^{*}$ satisfies the quantitative ordering property (QOP) if there is an isomorphism-invariant map that assigns to each structure $\boldsymbol{A}^{*}=\left\langle\boldsymbol{A},<_{\boldsymbol{A}}\right\rangle \in \mathcal{K}^{*}$ a real number $\rho\left(\boldsymbol{A}^{*}\right) \in[0,1]$ such that for every $\boldsymbol{A} \in \mathcal{K}^{*}$ and every $\epsilon>0$, there is a $\boldsymbol{B}=\boldsymbol{B}(\boldsymbol{A}, \epsilon) \in \mathcal{K}$ and a nonempty set of embeddings $E=E(\boldsymbol{A}, \epsilon)$ of $\boldsymbol{A}$ into $\boldsymbol{B}$ with the property that for each pair of $\mathcal{K}^{*}$-admissible orderings $<_{\boldsymbol{A}}$ of $\boldsymbol{A}$ and $<_{\boldsymbol{B}}$ of $\boldsymbol{B}$, the proportion of embeddings in $E$ that preserve $<_{\boldsymbol{A}}$ and $<_{\boldsymbol{B}}$ is equal to $\rho\left(\left\langle\boldsymbol{A},<_{\boldsymbol{A}}\right\rangle\right)$, within $\epsilon$.

There is also a slight variation of this property, which we denote by QOP*, that reads as follows: Let $\mathcal{K}$ be a Fraïssé class and $\mathcal{K}^{*}$ be an order expansion of $\mathcal{K}$. We say that $\mathcal{K}^{*}$ satisfies the $\boldsymbol{Q} \boldsymbol{O P} \boldsymbol{P}^{*}$ if there is an isomorphism-invariant map that assigns to each structure $\boldsymbol{A}^{*}=\left\langle\boldsymbol{A},<_{\boldsymbol{A}}\right\rangle \in \mathcal{K}^{*}$ a real number $\rho\left(\boldsymbol{A}^{*}\right) \in[0,1]$ such that for every $\boldsymbol{A}^{*}=\left\langle\boldsymbol{A},<_{\boldsymbol{A}}\right\rangle \in \mathcal{K}^{*}$ and every $\epsilon>0$, there is a $\boldsymbol{B}=\boldsymbol{B}\left(\boldsymbol{A}^{*}, \epsilon\right) \in \mathcal{K}$ and a nonempty set of embeddings $E=E\left(\boldsymbol{A}^{*}, \epsilon\right)$ of $\boldsymbol{A}$ into $\boldsymbol{B}$ with the property that for each $\mathcal{K}^{*}$-admissible ordering $<_{\boldsymbol{B}}$ of $\boldsymbol{B}$, the proportion of embeddings in $E$ that preserve $<_{\boldsymbol{A}}$ and $<_{\boldsymbol{B}}$ is equal to $\rho\left(\left\langle\boldsymbol{A},<_{\boldsymbol{A}}\right\rangle\right)$, within $\epsilon$.

The QOP implies the QOP*; for Hrushovski classes, they are equivalent by Theorem 13.3. Note that the QOP does not imply the ordering property, unless the function $\rho$ above is strictly positive.

We now have the following result, whose proof is related to that of Lemma 2.1. 
Proposition 11.1 Let $\mathcal{K}$ be a Fraïssé class and $\mathcal{K}^{*}$ be a Fraïssé class that is a reasonable order expansion of $\mathcal{K}$. Write $\boldsymbol{K}:=\operatorname{Flim}(\mathcal{K})$ and $\Gamma:=\operatorname{Aut}(\boldsymbol{K})$. If $\Gamma$ is amenable and the $Q O P^{*}$ holds for $\mathcal{K}^{*}$, then the $\Gamma$-flow $X_{\mathcal{K}^{*}}$ is uniquely ergodic. If moreover $\left(\mathcal{K}, \mathcal{K}^{*}\right)$ is an excellent pair, then $\Gamma$ is uniquely ergodic.

Proof. Since $\Gamma$ is amenable, the $\Gamma$-flow $X_{\mathcal{K}^{*}}$ has an invariant measure $\mu$. Let $\left(\mu_{\boldsymbol{A}}\right)_{\boldsymbol{A} \in \mathcal{K}}$ be the associated consistent random $\mathcal{K}^{*}$-ordering. For each $\left\langle\boldsymbol{A},<_{\boldsymbol{A}}\right\rangle \in \mathcal{K}^{*}$, we shall show that $\mu_{\boldsymbol{A}}\left(<_{\boldsymbol{A}}\right)=\rho\left(\left\langle\boldsymbol{A},<_{\boldsymbol{A}}\right\rangle\right)$, where $\rho$ comes from the QOP* ${ }^{*}$ this shows the uniqueness of $\mu$.

Fix such $\left\langle\boldsymbol{A},<_{\boldsymbol{A}}\right\rangle$ and $\epsilon>0$. Let $\boldsymbol{B}$ and $E$ be as in the definition of the QOP*. For each $f \in E$, we have

$$
\mu_{\boldsymbol{A}}\left(<_{\boldsymbol{A}}\right)=\mu_{f(\boldsymbol{A})}\left(f_{*}\left(<_{\boldsymbol{A}}\right)\right)=\sum\left\{\mu_{\boldsymbol{B}}\left(<_{\boldsymbol{B}}\right) ;\left\langle\boldsymbol{B},<_{\boldsymbol{B}}\right\rangle \in \mathcal{K}^{*}, f_{*}\left(<_{\boldsymbol{A}}\right) \subseteq<_{\boldsymbol{B}}\right\},
$$

so

$$
\begin{aligned}
|E| \cdot \mu_{\boldsymbol{A}}\left(<_{\boldsymbol{A}}\right) & =\sum_{f \in E} \sum\left\{\mu_{\boldsymbol{B}}\left(<_{\boldsymbol{B}}\right) ;\left\langle\boldsymbol{B},<_{\boldsymbol{B}}\right\rangle \in \mathcal{K}^{*}, f_{*}\left(<_{\boldsymbol{A}}\right) \subseteq<_{\boldsymbol{B}}\right\} \\
& =\sum_{\left\langle\boldsymbol{B},<_{\boldsymbol{B}}\right\rangle \in \mathcal{K}^{*}} \sum\left\{\mu_{\boldsymbol{B}}\left(<_{\boldsymbol{B}}\right) ; f \in E, f_{*}\left(<_{\boldsymbol{A}}\right) \subseteq<_{\boldsymbol{B}}\right\} \\
& =\sum_{\left\langle\boldsymbol{B},<_{\boldsymbol{B}}\right\rangle \in \mathcal{K}^{*}} \mu_{\boldsymbol{B}}\left(<_{\boldsymbol{B}}\right) \cdot\left|\left\{f \in E ; \quad f_{*}\left(<_{\boldsymbol{A}}\right) \subseteq<_{\boldsymbol{B}}\right\}\right|,
\end{aligned}
$$

and thus

$$
\mu_{\boldsymbol{A}}\left(<_{\boldsymbol{A}}\right)=\sum_{\left\langle B,<_{\boldsymbol{B}}\right\rangle \in \mathcal{K}^{*}} \frac{\left|\left\{f \in E ; f_{*}\left(<_{\boldsymbol{A}}\right) \subseteq<_{\boldsymbol{B}}\right\}\right|}{|E|} \cdot \mu_{\boldsymbol{B}}\left(<_{\boldsymbol{B}}\right) .
$$

Since $\sum_{\left\langle\boldsymbol{B},<_{\boldsymbol{B}}\right\rangle \in \mathcal{K}^{*}} \mu_{\boldsymbol{B}}\left(<_{\boldsymbol{B}}\right)=1$, this shows that $\left|\mu_{\boldsymbol{A}}\left(<_{\boldsymbol{A}}\right)-\rho\left(\left\langle\boldsymbol{A},<_{\boldsymbol{A}}\right\rangle\right)\right|<\epsilon$, and the proof is complete.

In Sections 2-6, we have seen that many excellent pairs $\left(\mathcal{K}, \mathcal{K}^{*}\right)$ satisfy the QOP and therefore the uniqueness of consistent random $\mathcal{K}^{*}$-admissible orderings. As a sample, we have the following result.

Theorem 11.2 The automorphism groups of the random $\mathcal{A}$-free hypergraph of a given type and the Urysohn space $\boldsymbol{U}_{S}$ are uniquely ergodic, but not compact nor extremely amenable.

We remark that the Urysohn space $\boldsymbol{U}$ without any restriction on distances, which is not a Fraïssé structure since it is uncountable, has an extremely amenable isometry group: see [Pe2]. 


\section{Hrushovski Structures}

Let $\boldsymbol{K}$ be a Hrushovski structure. Then there is a sequence $\Delta_{0} \subseteq \Delta_{1} \subseteq \cdots$ of compact subgroups of $\Gamma:=\operatorname{Aut}(\boldsymbol{K})$ with $\overline{\bigcup_{n} \Delta_{n}}=\Gamma$. We shall now prove a stronger version of this fact that will be used in the next section.

Proposition 12.1 Let $\boldsymbol{K}$ be a Hrushovski structure. Then we can find a sequence $\boldsymbol{A}_{0} \subseteq$ $\boldsymbol{A}_{1} \subseteq \cdots$ of finite substructures of $\boldsymbol{K}$ with $\boldsymbol{K}=\bigcup_{n} \boldsymbol{A}_{n}$ and a sequence of compact subgroups $\Delta_{0} \subseteq \Delta_{1} \subseteq \cdots$ of $\Gamma:=\operatorname{Aut}(\boldsymbol{K})$ with $\overline{\bigcup_{n} \Delta_{n}}=\Gamma$ such that for each $n, A_{n}$ is invariant under $\Delta_{n}$ and $g \in \Delta_{n} \mapsto g\left\lceil A_{n} \in \operatorname{Aut}\left(\boldsymbol{A}_{n}\right)\right.$ is a surjection from $\Delta_{n}$ onto $\operatorname{Aut}\left(\boldsymbol{A}_{n}\right)$.

Proof. Let $\Lambda_{0} \subseteq \Lambda_{1} \subseteq \cdots$ be compact subgroups of $\Gamma$ with $\overline{\bigcup_{n} \Lambda_{n}}=\Gamma$. Fix an enumeration $\left\{a_{0}, a_{1}, \ldots\right\}=K$. We shall construct recursively $\boldsymbol{A}_{n}, \Delta_{n}$ as above such that for each $n$, $\Lambda_{n} \subseteq \Delta_{n} \subseteq \Lambda_{n^{\prime}}$ for some $n^{\prime} \geq n$, and $A_{n} \supseteq\left\{a_{0}, \ldots, a_{n}\right\}$.

We take $\boldsymbol{A}_{0}$ to be a finite substructure of $\boldsymbol{K}$ that contains $a_{0}$ and is closed under $\Lambda_{0}$. Such exists by the compactness of $\Lambda_{0}$. Let $\operatorname{Aut}\left(\boldsymbol{A}_{0}\right)=\left\{\varphi_{1}, \ldots, \varphi_{k}\right\}$. Since $\bigcup_{n} \Lambda_{n}$ is dense in $\Gamma$, there are $f_{1}, \ldots, f_{k} \in \Lambda_{M}$ for some large $M \geq 1$ such that $f_{i}\left\lceil A_{0}=\varphi_{i}\right.$ for $1 \leq i \leq k$. Then $\Lambda_{0} \cup\left\{f_{1}, \ldots, f_{k}\right\} \subseteq \Lambda_{M}$. Put

$$
\Delta_{0}:=\overline{\left\langle\Lambda_{0} \cup\left\{f_{1}, \ldots, f_{k}\right\}\right\rangle},
$$

which is a compact subgroup of $\Lambda_{M}$ such that $\Lambda_{0} \subseteq \Delta_{0} \subseteq \Lambda_{0^{\prime}}$, where $0^{\prime}:=M$. Clearly $A_{0}$ is invariant under $\Delta_{0}$ and the restriction map from $\Delta_{0}$ to $\operatorname{Aut}\left(\boldsymbol{A}_{0}\right)$ is surjective.

Assume now $\boldsymbol{A}_{n}, \Delta_{n}$ have been constructed. To define $\boldsymbol{A}_{n+1}, \Delta_{n+1}$, we proceed as before. Let $\boldsymbol{A}_{n+1}$ be a finite substructure of $\boldsymbol{K}$ with $\boldsymbol{A}_{n} \subseteq \boldsymbol{A}_{n+1}$ such that $a_{n+1} \in A_{n+1}$ and $A_{n+1}$ is invariant under $\Lambda_{m}$, where $m:=\max \left\{n+1, n^{\prime}\right\}$, so that also $\Lambda_{m} \supseteq \Delta_{n} \cup \Lambda_{n+1}$. Let $\operatorname{Aut}\left(\boldsymbol{A}_{n+1}\right)=\left\{\psi_{1}, \ldots, \psi_{\ell}\right\}$. As before, there is $N \geq m$ and $f_{1}, \ldots, f_{\ell} \in \Lambda_{N}$ such that $f_{i} \uparrow A_{n+1}=\psi_{i}$ for $1 \leq i \leq \ell$. Put

$$
\Delta_{n+1}:=\overline{\left\langle\Lambda_{m} \cap\left\{f_{1}, \ldots, f_{\ell}\right\}\right\rangle} \subseteq \Lambda_{N},
$$

so that $\Delta_{n+1}$ is compact, $\Delta_{n+1} \supseteq \Delta_{n} \cup \Lambda_{n+1}$, and $\Delta_{n+1} \subseteq \Lambda_{(n+1)^{\prime}}$, where $(n+1)^{\prime}=N$. Finally, $A_{n+1}$ is invariant under $\Delta_{n+1}$ and the restriction map from $\Delta_{n+1}$ to $\operatorname{Aut}\left(\boldsymbol{A}_{n+1}\right)$ is surjective.

Let $\boldsymbol{K}$ be a Hrushovski structure. A sequence $\left\langle\left(\boldsymbol{A}_{n}, \Delta_{n}\right) ; n \geq 0\right\rangle$ as in the previous theorem will be called characteristic.

\section{Equivalence of Unique Ergodicity and the QOP for Hrushovski Structures}

We shall now consider unique ergodicity in the context of Hrushovski classes. Note first that if $\mathcal{K}$ is a Hrushovski class and $\boldsymbol{K}=\mathrm{Flim}(\mathcal{K})$, there is a sequence of finite substructures 
$\boldsymbol{A}_{0} \subseteq \boldsymbol{A}_{1} \subseteq \cdots \subseteq \boldsymbol{K}$ such that $\bigcup_{n} \boldsymbol{A}_{n}=\boldsymbol{K}$ and every isomorphism between substructures of $\boldsymbol{A}_{n}$ extends to an automorphism of $\boldsymbol{A}_{n+1}$. We now have for every such sequence $\left\langle\boldsymbol{A}_{n}\right\rangle$ :

Proposition 13.1 Assume that the Hrushovski class $\mathcal{K}$ admits a companion $\mathcal{K}^{*}$. Let $\boldsymbol{K}:=$ $\operatorname{Flim}(\mathcal{K})$. For each finite $\boldsymbol{A} \subseteq \boldsymbol{K}$ and each $\mathcal{K}^{*}$-admissible ordering $<$ on $\boldsymbol{A}$, let

$$
\mu_{n}(<):=\frac{\left|\left\{<_{n} ;\left\langle\boldsymbol{A}_{n},<_{n}\right\rangle \in \mathcal{K}^{*},<\subseteq<_{n}\right\}\right|}{\left|\left\{<_{n} ;\left\langle\boldsymbol{A}_{n},<_{n}\right\rangle \in \mathcal{K}^{*}\right\}\right|}
$$

for any $n$ such that $\boldsymbol{A} \subseteq \boldsymbol{A}_{n}$. Let $\mathcal{U}$ be a non-principal ultrafilter on $\mathbb{N}$ and put

$$
\mu\left(N_{\boldsymbol{A},<}\right):=\mu(<):=\lim _{n \rightarrow \mathcal{U}} \mu_{n}(<) .
$$

Then $\mu$ is a $\Gamma$-invariant measure on $X_{\mathcal{K}^{*}}$, where $\Gamma:=\operatorname{Aut}(\boldsymbol{K})$.

Proof. It is easy to check that $\mu$ defines a measure. We next check its $\Gamma$-invariance. Let $g \in \Gamma$ and let $g(\langle\boldsymbol{A},<\rangle)=:\left\langle\boldsymbol{B},<^{\prime}\right\rangle$ in order to check that $\mu(<)=\mu\left(<^{\prime}\right)$. Let $n_{0}$ be large enough so that $\boldsymbol{A}, \boldsymbol{B} \subseteq \boldsymbol{A}_{n_{0}}$. Let $n>n_{0}$. Then $g$ restricted to $\boldsymbol{A}$ is an isomorphism between substructures of $\boldsymbol{A}_{n_{0}}$, so $g$ extends to an automorphism of $\boldsymbol{A}_{n}$. Clearly $\mu_{n}(<)=\mu_{n}\left(<^{\prime}\right)$, so $\mu(<)=\mu\left(<^{\prime}\right)$.

This gives us the following formula for every such sequence $\left\langle\boldsymbol{A}_{n}\right\rangle$ in the case of unique ergodicity.

Theorem 13.2 Let $\mathcal{K}$ be a Hrushovski class that admits a companion $\mathcal{K}^{*}$. Let $\boldsymbol{K}:=\operatorname{Flim}(\mathcal{K})$ and $\Gamma:=\operatorname{Aut}(\boldsymbol{K})$. If $\Gamma$ is uniquely ergodic, then the unique $\Gamma$-invariant measure $\mu$ on $X_{\mathcal{K}^{*}}$ is given by

$$
\mu\left(N_{\boldsymbol{A},<}\right):=\lim _{n \rightarrow \infty} \mu_{n}(<) .
$$

Proof. This follows from the preceding proposition and the fact that for any bounded sequence $\left\langle a_{n}\right\rangle$ of reals, $\lim _{n} a_{n}=a$ iff for every non-principal ultrafilter $\mathcal{U}$ on $\mathbb{N}$, we have $\lim _{n \rightarrow \mathcal{U}} a_{n}=a$.

We shall next see that for Hrushovski classes $\mathcal{K}$ admitting a companion $\mathcal{K}^{*}$, unique ergodicity for $\Gamma:=\operatorname{Aut}(\boldsymbol{K})$, with $\boldsymbol{K}:=\operatorname{Flim}(\mathcal{K})$, is actually equivalent to the quantitative ordering property for $\mathcal{K}^{*}$.

Theorem 13.3 Let $\mathcal{K}$ be a Hrushovski class, $\mathcal{K}^{*}$ be a Fraïsé class that is a reasonable order expansion of $\mathcal{K}$, and let $\boldsymbol{K}:=\operatorname{Flim}(\mathcal{K})$ and $\Gamma:=\operatorname{Aut}(\boldsymbol{K})$. Then the following are equivalent:

(i) The $\Gamma$-flow $X_{\mathcal{K}^{*}}$ is uniquely ergodic.

(ii) There is an isomorphism-invariant map $\rho: \mathcal{K}^{*} \rightarrow[0,1]$ such that for every $\boldsymbol{A} \in \mathcal{K}$ and every $\epsilon>0$, there is $\boldsymbol{B} \in \mathcal{K}$ with $\boldsymbol{B} \supseteq \boldsymbol{A}$ such that for every $\left\langle\boldsymbol{A},<_{\boldsymbol{A}}\right\rangle \in \mathcal{K}^{*}$ and $\left\langle\boldsymbol{B},<_{\boldsymbol{B}}\right\rangle \in \mathcal{K}^{*}$, the proportion of automorphisms $\pi$ of $\boldsymbol{B}$ such that $\pi_{*}\left(<_{\boldsymbol{A}}\right) \subseteq<_{\boldsymbol{B}}$ is equal to $\rho\left(\left\langle\boldsymbol{A},<_{\boldsymbol{A}}\right\rangle\right)$, within $\epsilon$. 
(iii) There is an isomorphism-invariant map $\rho: \mathcal{K}^{*} \rightarrow[0,1]$ such that for every $\boldsymbol{A}^{*}=$ $\left\langle\boldsymbol{A},<_{\boldsymbol{A}}\right\rangle \in \mathcal{K}^{*}$ and every $\epsilon>0$, there is $\boldsymbol{B} \in \mathcal{K}$ with $\boldsymbol{B} \supseteq \boldsymbol{A}$ such that for every $\left\langle\boldsymbol{B},<_{\boldsymbol{B}}\right\rangle \in \mathcal{K}$, the proportion of automorphisms $\pi$ of $\boldsymbol{B}$ such that $\pi_{*}\left(<_{\boldsymbol{A}}\right) \subseteq<_{\boldsymbol{B}}$ is equal to $\rho\left(\left\langle\boldsymbol{A},<_{\boldsymbol{A}}\right\rangle\right)$, within $\epsilon$.

(iv) (QOP) There is an isomorphism-invariant map $\rho: \mathcal{K}^{*} \rightarrow[0,1]$ such that for every $\boldsymbol{A} \in \mathcal{K}$ and each $\epsilon>0$, there is a $\boldsymbol{B} \in \mathcal{K}$ and a nonempty set of embeddings $E$ of $\boldsymbol{A}$ into $\boldsymbol{B}$ with the property that for each $\mathcal{K}^{*}$-admissible ordering $<_{\boldsymbol{A}}$ of $\boldsymbol{A}$ and each $\mathcal{K}^{*}$ admissible ordering $<_{\boldsymbol{B}}$ of $\boldsymbol{B}$, the proportion of embeddings in $E$ that preserve $<_{\boldsymbol{A}},<_{\boldsymbol{B}}$ is equal to $\rho\left(\left\langle\boldsymbol{A},<_{\boldsymbol{A}}\right\rangle\right)$, within $\epsilon$.

(v) $\left(Q O P^{*}\right)$ There is an isomorphism-invariant map $\rho: \mathcal{K}^{*} \rightarrow[0,1]$ such that for every $\boldsymbol{A}^{*}=\left\langle\boldsymbol{A},<_{\boldsymbol{A}}\right\rangle \in \mathcal{K}^{*}$ and each $\epsilon>0$, there is a $\boldsymbol{B} \in \mathcal{K}$ and a nonempty set of embeddings $\boldsymbol{E}$ of $\boldsymbol{A}$ into $\boldsymbol{B}$ with the property that for each $\mathcal{K}^{*}$-admissible ordering $<_{\boldsymbol{B}}$ of $\boldsymbol{B}$, the proportion of embeddings in $E$ that preserve $<_{\boldsymbol{A}},<_{\boldsymbol{B}}$ is equal to $\rho\left(\left\langle\boldsymbol{A},<_{\boldsymbol{A}}\right\rangle\right)$, within $\epsilon$.

Moreover, if $\mathcal{K}^{*}$ has the $\mathrm{OP}$, then $(i)-(v)$ are equivalent to

(vi) The same as (ii), but with $\rho$ strictly positive.

(vii) The same as (iii), but with $\rho$ strictly positive.

(viii) The same as (iv), but with $\rho$ strictly positive.

(ix) The same as (v), but with $\rho$ strictly positive.

Finally, if $\left(\mathcal{K}, \mathcal{K}^{*}\right)$ is an excellent pair, then $(i)-(i x)$ are equivalent to

(x) $\Gamma$ is uniquely ergodic.

Proof. (i) $\Rightarrow$ (ii). Let $\mu$ be the unique invariant measure for the $\Gamma$-flow $X_{\mathcal{K}^{*}}$. Put

$$
\rho(\langle\boldsymbol{A},<\rangle):=\mu\left(N_{\boldsymbol{A},<}\right)
$$

for any $\boldsymbol{A} \in \mathcal{K}, \boldsymbol{A} \subseteq \boldsymbol{K}$ and $\langle\boldsymbol{A},<\rangle \in \mathcal{K}^{*}$. This extends in an obvious way to an isomorphisminvariant map on all of $\mathcal{K}^{*}$, also denoted by $\rho$ (since each $\boldsymbol{A} \in \mathcal{K}$ has an isomorphic copy contained in $\boldsymbol{K}$ and any two such copies are isomorphic via an automorphism of $\boldsymbol{K}$ ).

Consider now a characteristic sequence $\left\langle\left(\boldsymbol{A}_{n}, \Delta_{n}\right) ; n \geq 0\right\rangle$ as in Section 12. Write $\mu_{n}$ for the Haar measure of $\Delta_{n}$. Then

$$
A_{n}(f)(x):=\int_{\Delta_{n}} f\left(g^{-1} \cdot x\right) d \mu_{n}(g)
$$

converges uniformly to $\int f d \mu$ for every $f \in C\left(X_{\mathcal{K}^{*}}\right)$ by Theorem 8.2. For $\boldsymbol{A}^{*}:=\langle\boldsymbol{A},<\rangle \in \mathcal{K}^{*}$ with $\boldsymbol{A} \subseteq \boldsymbol{K}$, denote by $\mathbf{1}_{\boldsymbol{A}^{*}}$ the indicator function of the set $\left\{\prec \in X_{\mathcal{K}^{*}} ;<\subseteq \prec\right\}$. Then $\mathbf{1}_{\boldsymbol{A}^{*}}$ is continuous, so

$$
\int_{\Delta_{n}} \mathbf{1}_{\boldsymbol{A}^{*}}\left(g^{-1} \cdot x\right) d \mu_{n}(g) \rightarrow \int \mathbf{1}_{\boldsymbol{A}^{*}} d \mu=\rho(\boldsymbol{A},<)
$$


uniformly. For $x=\prec \in X_{\mathcal{K}^{*}}$, the left-hand side is

$$
\int_{\Delta_{n}} \mathbf{1}_{\boldsymbol{A}^{*}}\left(g^{-1} \cdot x\right) d \mu_{n}(g)=\mu_{n}\left(\left\{g \in \Delta_{n} ; g_{*}(<) \subseteq \prec\right\}\right) .
$$

Find $n_{\boldsymbol{A}^{*}}$ large enough so that

$$
\left|\mu_{n}\left(\left\{g \in \Delta_{n} ; g_{*}(<) \subseteq \prec\right\}\right)-\rho\left(\boldsymbol{A}^{*}\right)\right|<\epsilon
$$

for all $\prec \in X_{\mathcal{K}^{*}}$ and $n \geq n_{\boldsymbol{A}^{*}}$. Since there are only finitely many $\langle\boldsymbol{A},<\rangle \in \mathcal{K}^{*}$ (where $\boldsymbol{A}$ is fixed), we can find $N$ large enough so that $\boldsymbol{A} \subseteq \boldsymbol{A}_{N}$ and (3) holds for all $<$ with $\boldsymbol{A}^{*}=\langle\boldsymbol{A},<\rangle \in \mathcal{K}^{*}, \prec \in X_{\mathcal{K}^{*}}$, and $n \geq N$. Take then $\boldsymbol{B}:=\boldsymbol{A}_{N}$. Let $\langle\boldsymbol{A},<\rangle \in \mathcal{K}^{*}$, let $<^{\prime}$ be $\mathcal{K}^{*}$-admissible for $\boldsymbol{B}$, and let $\prec \in X_{\mathcal{K}^{*}}$ extend $<^{\prime}$. We only have to check that

$$
\mu_{N}\left(\left\{g \in \Delta_{N} ; g_{*}(<) \subseteq \prec\right\}\right)=\frac{\left|\left\{\pi \in \operatorname{Aut}(\boldsymbol{B}) ; \pi_{*}(<) \subseteq \prec\right\}\right|}{|\operatorname{Aut}(\boldsymbol{B})|}
$$

Indeed, let $\varphi: \Delta_{N} \rightarrow \operatorname{Aut}(\boldsymbol{B})$ be the epimorphism $\varphi(g):=g \uparrow B$. Then

$$
\begin{aligned}
\mu_{N}\left(\left\{g \in \Delta_{N} ; g_{*}(<) \subseteq \prec\right\}\right) & =\mu_{N}\left(\left\{g \in \Delta_{N} ; \varphi(g)_{*}(<) \subseteq \prec\right\}\right) \\
& =\mu_{N}(\operatorname{ker}(\varphi)) \cdot\left|\left\{\pi \in \operatorname{Aut}(\boldsymbol{B}) ; \pi_{*}(<) \subseteq \prec\right\}\right| .
\end{aligned}
$$

But clearly $\mu_{N}(\operatorname{ker}(\varphi))=\frac{1}{|\operatorname{Aut}(\boldsymbol{B})|}$, so we are done.

(ii) $\Rightarrow$ (iii) is obvious.

(iii) $\Rightarrow$ (i). This is similar to the proof of Proposition 11.1. Since $\Gamma$ is amenable, the $\Gamma$-flow $X_{\mathcal{K}^{*}}$ has an invariant measure. Let $\mu$ be any such measure. For each $\left\langle\boldsymbol{A},{ }_{\boldsymbol{A}}\right\rangle \in \mathcal{K}^{*}$ with $\boldsymbol{A} \subseteq \boldsymbol{K}$, we shall show that $\mu\left(N_{\boldsymbol{A},<_{\boldsymbol{A}}}\right)=\rho\left(\left\langle\boldsymbol{A},<_{\boldsymbol{A}}\right\rangle\right)$, which shows the uniqueness of $\mu$.

Fix such $\left\langle\boldsymbol{A},<_{\boldsymbol{A}}\right\rangle$ and $\epsilon>0$. Let $\boldsymbol{B}$ be as in (iii). For each $\pi \in \operatorname{Aut}(\boldsymbol{B})$, we have

$$
\mu\left(N_{\boldsymbol{A},<_{\boldsymbol{A}}}\right)=\sum\left\{\mu\left(N_{\boldsymbol{B},<_{\boldsymbol{B}}}\right) ;\left\langle\boldsymbol{B},<_{\boldsymbol{B}}\right\rangle \in \mathcal{K}^{*}, \pi_{*}\left(<_{\boldsymbol{A}}\right) \subseteq<_{\boldsymbol{B}}\right\}
$$

since $\mu\left(N_{\boldsymbol{A},<_{\boldsymbol{A}}}\right)=\mu\left(N_{\pi_{*}(\boldsymbol{A}), \pi_{*}\left(<_{\boldsymbol{A}}\right)}\right)$ and $N_{\pi_{*}(\boldsymbol{A}), \pi_{*}\left(<_{\boldsymbol{A}}\right)}$ is the disjoint union of the sets $N_{\boldsymbol{B},<_{\boldsymbol{B}}}$ with $\left\langle\boldsymbol{B},<_{\boldsymbol{B}}\right\rangle \in \mathcal{K}^{*}$ and $\pi_{*}\left(<_{\boldsymbol{A}}\right) \subseteq<_{\boldsymbol{B}}$. So

$$
\begin{aligned}
|\operatorname{Aut}(\boldsymbol{B})| \cdot \mu\left(N_{\boldsymbol{A},<_{\boldsymbol{A}}}\right) & =\sum_{\pi \in \operatorname{Aut}(\boldsymbol{B})} \mu\left(N_{\left.\boldsymbol{A},<_{\boldsymbol{A}}\right)}\right. \\
& =\sum_{\pi \in \operatorname{Aut}(\boldsymbol{B})} \sum\left\{\mu\left(N_{\boldsymbol{B},<_{\boldsymbol{B}}}\right) ;\left\langle\boldsymbol{B},<_{\boldsymbol{B}}\right\rangle \in \mathcal{K}^{*}, \pi_{*}\left(<_{\boldsymbol{A}}\right) \subseteq<_{\boldsymbol{B}}\right\} \\
& =\sum_{\left\langle\boldsymbol{B},<_{\boldsymbol{B}}\right\rangle \in \mathcal{K}^{*}} \sum\left\{\mu\left(N_{\boldsymbol{B},<_{\boldsymbol{B}}}\right) ; \pi \in \operatorname{Aut}(\boldsymbol{B}), \pi_{*}\left(<_{\boldsymbol{A}}\right) \subseteq<_{\boldsymbol{B}}\right\} \\
& =\sum_{\left\langle\boldsymbol{B},<_{\boldsymbol{B}}\right\rangle \in \mathcal{K}^{*}} \mu\left(N_{\boldsymbol{B},<_{\boldsymbol{B}}}\right) \cdot\left|\left\{\pi \in \operatorname{Aut}(\boldsymbol{B}) ; \pi_{*}\left(<_{\boldsymbol{A}}\right) \subseteq<_{\boldsymbol{B}}\right\}\right|,
\end{aligned}
$$


whence

$$
\mu\left(N_{\boldsymbol{A},<_{\boldsymbol{A}}}\right)=\sum_{\left\langle\boldsymbol{B},<_{\boldsymbol{B}}\right\rangle \in \mathcal{K}^{*}} \frac{\left|\left\{\pi \in \operatorname{Aut}(\boldsymbol{B}) ; \pi_{*}\left(<_{\boldsymbol{A}}\right) \subseteq<_{\boldsymbol{B}}\right\}\right|}{|\operatorname{Aut}(\boldsymbol{B})|} \cdot \mu\left(N_{\boldsymbol{B},<_{\boldsymbol{B}}}\right) .
$$

Since $\sum_{\left\langle\boldsymbol{B},<_{\boldsymbol{B}}\right\rangle \in \mathcal{K}^{*}} \mu\left(N_{\boldsymbol{B},<_{\boldsymbol{B}}}\right)=1$, this shows that we have $\left|\mu\left(N_{\boldsymbol{A},<_{\boldsymbol{A}}}\right)-\rho\left(\left\langle\boldsymbol{A},<_{\boldsymbol{A}}\right\rangle\right)\right|<\epsilon$.

(ii) $\Rightarrow$ (iv). Let $\rho$ be as in (ii). Given $\boldsymbol{A}$ and $\epsilon>0$, let $\boldsymbol{B}$ again be as in (ii). Let $E$ consist of the restrictions to $\boldsymbol{A}$ of all the automorphisms of $\boldsymbol{B}$. For $f \in E$, let $A_{f}:=\{\pi \in$ $\operatorname{Aut}(\boldsymbol{B}) ; \pi\lceil A=f\}$. It is clearly enough to show that $\left|A_{f}\right|$ is independent of $f$. So take $f_{1}, f_{2} \in E$ and fix $\pi_{1} \in A_{f_{1}}, \pi_{2} \in A_{f_{2}}$. It is enough to show that for $\sigma:=\pi_{2} \circ \pi_{1}^{-1}$, we have $\sigma \circ A_{f_{1}} \subseteq A_{f_{2}}$. Indeed, let $\pi \in A_{f_{1}}$. Then for $a \in A$, we have $\sigma \circ \pi(a)=\sigma\left(f_{1}(a)\right)=$ $\sigma\left(\pi_{1}(a)\right)=\pi_{2}(a)=f_{2}(a)$, i.e., $\sigma \circ \pi \in A_{f_{2}}$.

(iv) $\Rightarrow(\mathrm{v})$ is obvious.

$(\mathrm{v}) \Rightarrow(\mathrm{i})$. This follows by Proposition 11.1.

If $\mathcal{K}^{*}$ satisfies the OP, then $X_{\mathcal{K}^{*}}$ is a minimal $\Gamma$-flow and thus the (closed) support of any invariant measure is equal to $X_{\mathcal{K}^{*}}$, whence in all of (ii)-(v), we may take $\rho$ to be strictly positive, i.e., (vi)-(ix) hold.

Finally, the equivalence with (x) follows from Proposition 8.1 and Theorem 9.1.

Remark 13.4 In Theorem 13.3, consider the following strengthening of (ii):

(ii)' There is an isomorphism-invariant map $\rho: \mathcal{K}^{*} \rightarrow[0,1]$ such that for every $\boldsymbol{A} \in \mathcal{K}$ and $\epsilon>0$, there is $\boldsymbol{B} \in \mathcal{K}$ with $\boldsymbol{B} \supseteq \boldsymbol{A}$ such that for $\boldsymbol{A}_{0} \subseteq \boldsymbol{A},\left\langle\boldsymbol{A}_{0},<_{0}\right\rangle \in \mathcal{K}^{*}$, and every $\mathcal{K}^{*}$-admissible ordering $<_{\boldsymbol{B}}$ for $\boldsymbol{B}$, we have

$$
\left|\frac{\left|\left\{\pi \in \operatorname{Aut}(\boldsymbol{B}) ; \pi_{*}\left(<_{0}\right) \subseteq<_{\boldsymbol{B}}\right\}\right|}{|\operatorname{Aut}(\boldsymbol{B})|}-\rho\left(\left\langle\boldsymbol{A}_{0},<_{0}\right\rangle\right)\right|<\epsilon .
$$

It is easy to check that the proof of Theorem 13.3 also shows that for a Hrushovski class $\mathcal{K}$, (ii)' is equivalent to (ii). However, we can see, without assuming that $\mathcal{K}$ is Hrushovski, that (ii) ${ }^{\prime} \Rightarrow(\mathrm{i})$ and thus when $\left(\mathcal{K}, \mathcal{K}^{*}\right)$ is an excellent pair, (ii) $)^{\prime}$ implies that $\Gamma:=\operatorname{Aut}(\boldsymbol{K})$ is uniquely ergodic.

We shall check that $\mu_{\boldsymbol{A}}(<):=\rho(\boldsymbol{A},<)$ for $\langle\boldsymbol{A},<\rangle \in \mathcal{K}^{*}$ is a consistent random $\mathcal{K}^{*}$ admissible ordering on $\mathcal{K}$. This shows that the $\Gamma$-flow $X_{\mathcal{K}^{*}}$ admits an invariant measure and the argument in Theorem 13.3 (iii) $\Rightarrow$ (i) shows that it is uniquely ergodic.

First note that

$$
\forall \boldsymbol{A} \in \mathcal{K} \quad \sum\left\{\rho\left(\boldsymbol{A}^{*}\right) ; \boldsymbol{A}^{*}\lceil L=\boldsymbol{A}\}=1,\right.
$$

since (ii)' implies that for all $\epsilon$, this sum is equal to 1 within $|A| ! \epsilon$.

Next fix $\boldsymbol{A}_{0} \subseteq \boldsymbol{A}$ in $\mathcal{K}$ and $\left(\boldsymbol{A}_{0},<_{0}\right) \in \mathcal{K}^{*}$ in order to show that

$$
\rho\left(\left\langle\boldsymbol{A}_{0},<_{0}\right\rangle\right)=\sum\left\{\rho(\boldsymbol{A},<) ;\langle\boldsymbol{A},<\rangle \in \mathcal{K}^{*},<_{0} \subseteq<\right\} .
$$

Let $\epsilon>0$ and let $\boldsymbol{B}$ be as in (ii)'. 
Note that for $\left\langle\boldsymbol{B},<_{\boldsymbol{B}}\right\rangle \in \mathcal{K}^{*}$,

$$
\begin{aligned}
\left\{\pi \in \operatorname{Aut}(\boldsymbol{B}) ; \pi_{*}\left(<_{0}\right) \subseteq<_{\boldsymbol{B}}\right\} & =\left\{\pi \in \operatorname{Aut}(\boldsymbol{B}) ;<_{0} \subseteq \pi^{*}\left(<_{\boldsymbol{B}}\right)\right\} \\
& =\bigsqcup_{\substack{\left\langle\boldsymbol{A},<>\in \mathcal{K}^{*} \\
<<\subseteq<\right.}}\left\{\pi \in \operatorname{Aut}(\boldsymbol{B}) ; \pi_{*}(<) \subseteq<_{\boldsymbol{B}}\right\},
\end{aligned}
$$

so

$$
\begin{gathered}
\frac{\left|\left\{\pi \in \operatorname{Aut}(\boldsymbol{B}) ; \pi_{*}\left(<_{0}\right) \subseteq<_{\boldsymbol{B}}\right\}\right|}{|\operatorname{Aut}(\boldsymbol{B})|}= \\
\sum\left\{\frac{\left|\left\{\pi \in \operatorname{Aut}(\boldsymbol{B}) ; \pi_{*}(<) \subseteq<_{\boldsymbol{B}}\right\}\right|}{|\operatorname{Aut}(\boldsymbol{B})|} ;\langle\boldsymbol{A},<\rangle \in \mathcal{K}^{*},<_{0} \subseteq<\right\} .
\end{gathered}
$$

Let

$$
a:=\sum\left\{\rho(\langle\boldsymbol{A},<\rangle) ;\langle\boldsymbol{A},<\rangle \in \mathcal{K}^{*},<_{0} \subseteq<\right\}
$$

and

$$
N:=\left|\left\{<;\langle\boldsymbol{A},<\rangle \in \mathcal{K}^{*},<_{0} \subseteq<\right\}\right| .
$$

Then

$$
a-N \epsilon<(*)<a+N \epsilon .
$$

Thus

$$
\left|\frac{\left|\left\{\pi \in \operatorname{Aut}(\boldsymbol{B}) ; \pi_{*}\left(<_{0}\right) \subseteq<_{\boldsymbol{B}}\right\}\right|}{|\operatorname{Aut}(\boldsymbol{B})|}-\sum\left\{\rho(\langle\boldsymbol{A},<\rangle) ;\langle\boldsymbol{A},<\rangle \in \mathcal{K}^{*},<_{0} \subseteq<\right\}\right|<N \epsilon .
$$

But also

$$
\left|\frac{\left|\left\{\pi \in \operatorname{Aut}(\boldsymbol{B}) ; \pi_{*}\left(<_{0}\right) \subseteq<_{\boldsymbol{B}}\right\}\right|}{|\operatorname{Aut}(\boldsymbol{B})|}-\rho\left(\left\langle\boldsymbol{A}_{0},<_{0}\right\rangle\right)\right|<\epsilon,
$$

so, taking $\epsilon \rightarrow 0$, we see that

$$
\rho\left(\boldsymbol{A}_{0},<_{0}\right)=\sum\left\{\rho(\langle\boldsymbol{A},<\rangle) ;\langle\boldsymbol{A},<\rangle \in \mathcal{K}^{*},<_{0} \subseteq<\right\} .
$$

A similar remark holds if we replace (iv) by the analogous (iv)'.

\section{The Support of the Unique Measure}

We shall show here that in certain situations where unique ergodicity holds, the unique measure is supported by a single orbit, which is actually comeager.

We first discuss the notion of generic point and orbit. If $\Gamma$ is a topological group that acts continuously on a topological space $X$, we say that $x \in X$ is generic if its orbit $\Gamma \cdot x$ is comeager. In this case, we also say that $\Gamma \cdot x$ is a generic orbit. Clearly there is at most one generic orbit in any Baire space $X$. We first note the following general fact: 
Proposition 14.1 Let $\Gamma$ be a Polish group acting continuously on topological spaces $X$ and $Y$ that are Hausdorff and Baire. Assume that the action of $\Gamma$ on $X$ is minimal and $\pi: X \rightarrow Y$ is a continuous surjective $\Gamma$-map. If $x_{0}$ is a generic point for $X$, then $\pi\left(x_{0}\right)$ is a generic point for $Y$.

Proof. We use arguments similar to those in Appendix A of Melleray and Tsankov [MT], although we need to exercise extra care because of our more general context.

Let $y_{0}:=\pi\left(x_{0}\right)$. First notice that $\Gamma \cdot y_{0}$ is dense in $Y$. We next verify that $\Gamma \cdot y_{0}$ has the Baire Property in $Y$. By the Nikodým Theorem (see [K, 29.14]), it is enough to show that $\Gamma \cdot y_{0}$ can be obtained via the Souslin operation $\mathcal{A}$ applied to closed sets in $Y$, i.e., can be written in the form $\mathcal{A}_{s} F_{s}:=\left\{y \in Y ; \exists \alpha \in \mathcal{N} \forall n\left(y \in F_{\alpha \mid n}\right)\right\}$, where $\mathcal{N}:=\mathbb{N}^{\mathbb{N}}$ is the Baire space and $\left(F_{s}\right)_{s \in \mathbb{N}<\mathbb{N}}$ is a family of closed sets indexed by the set of finite sequences from $\mathbb{N}$. To exhibit such a representation, let $\rho: \mathcal{N} \rightarrow \Gamma$ be a continuous surjection and let $f: \mathcal{N} \rightarrow Y$ be defined by $f(\alpha):=\rho(\alpha) \cdot y_{0}$. Clearly $f$ is continuous. Let $\mathcal{N}_{s}:=\{\alpha \in \mathcal{N} ; s \subseteq \alpha\}$, for $s \in \mathbb{N}^{<N}$, be the basic open sets in $\mathcal{N}$. Put $F_{s}:=\overline{f\left[\mathcal{N}_{s}\right]}$. Then it is easy to see that $\Gamma \cdot y_{0}=\mathcal{A}_{s} F_{s}$.

It follows that if $\Gamma \cdot y_{0}$ is not meager, it must be comeager (see $[\mathrm{K}, 8.46]$ ). That is, it suffices to show that $\Gamma \cdot y_{0}$ is not meager. Now if it were meager, then there would be a sequence $\left\langle V_{n}\right\rangle$ of dense open sets in $Y$ with $\bigcap_{n} V_{n} \cap \Gamma \cdot y_{0}=\emptyset$. This would imply that $\bigcap_{n} \pi^{-1}\left(V_{n}\right) \cap \Gamma \cdot x_{0}=\emptyset$, so it is enough to show that if $V \subseteq Y$ is dense and open in $Y$, then $\pi^{-1}(V)$ is dense in $X$. To show this, let $W \subseteq X$ be nonempty and open. Let $\Gamma_{0}$ be a countable dense subgroup of $\Gamma$. By the minimality of $X$, we have $\Gamma_{0} \cdot W=X$, whence $\Gamma_{0} \cdot \pi(W)=Y$. Then $\pi(W)$ is not meager in $Y$, thus $\pi(W) \cap V \neq \emptyset$, so $W \cap \pi^{-1}(V) \neq \emptyset$. $\dashv$

Specializing to the case of $\Gamma$-flows, we then have the following:

Corollary 14.2 Let $\Gamma$ be a Polish group. If the universal minimal flow of $\Gamma$ has a generic point, then so does every minimal flow of $\Gamma$.

Let us say that a Polish group $\Gamma$ has the generic point property if every minimal $\Gamma$-flow has a generic point. We note now the following:

Proposition 14.3 Let $\left(\mathcal{K}, \mathcal{K}^{*}\right)$ be an excellent pair. Let $\boldsymbol{K}:=\operatorname{Flim}(\mathcal{K})$ and $\Gamma:=\operatorname{Aut}(\boldsymbol{K})$. Then $\Gamma$ has the generic point property.

Proof. By the previous corollary, it is enough to show that $X_{\mathcal{K}^{*}}$ has a generic point. Let $\boldsymbol{K}^{*}:=\left\langle\boldsymbol{K},<^{*}\right\rangle$ be the Fraïssé limit of $\mathcal{K}^{*}$. Clearly the $\Gamma$-orbit of $<^{*}$ consists of all $<$ in $X_{\mathcal{K}^{*}}$ such that $\langle\boldsymbol{K},<\rangle \cong\left\langle\boldsymbol{K},<^{*}\right\rangle$, i.e., all $\langle\boldsymbol{K},<\rangle$ that are, up to isomorphism, the Fraïssé limit of $\mathcal{K}^{*}$. These are characterized by the following two properties (see [Ho, 7.1.4]):

(i) $\operatorname{Age}(\langle\boldsymbol{K},<\rangle)=\mathcal{K}^{*}$;

(ii) Given $\boldsymbol{A}^{*} \subseteq \boldsymbol{B}^{*}$ in $\mathcal{K}^{*}$ and an embedding $\pi: \boldsymbol{A}^{*} \rightarrow\langle\boldsymbol{K},<\rangle$, there is an embedding $\rho: \boldsymbol{B}^{*} \rightarrow\langle\boldsymbol{K},<\rangle$ extending $\pi$.

Since property (i) is true for all $<\in X_{\mathcal{K}^{*}}$, this orbit consists of all $<\in X_{\mathcal{K}^{*}}$ that satisfy condition (ii), and this is clearly a $G_{\delta}$ subset of $X_{\mathcal{K}^{*}}$. It is also dense by [KPT, Section 7]. $\dashv$ 
We now have the following result.

Theorem 14.4 Let $\left(\mathcal{K}, \mathcal{K}^{*}\right)$ be an excellent pair with $\mathcal{K}^{*}=\mathcal{K} * \mathcal{L O}$. Let $\boldsymbol{K}:=\operatorname{Flim}(\mathcal{K})$ and $\Gamma:=\operatorname{Aut}(\boldsymbol{K})$. Then the uniform measure on $X_{\mathcal{K}^{*}}$ is supported by the generic orbit. In particular, if $\Gamma$ is uniquely ergodic, then the unique measure in each minimal flow is supported by the generic orbit.

Proof. Since $\mathcal{K}^{*}=\mathcal{K} * \mathcal{L} \mathcal{O}$, it is clear that $X_{\mathcal{K}^{*}}=\mathrm{LO}(K)$ is the space of all linear orderings on $K$. Let $\boldsymbol{K}^{*}:=\left\langle\boldsymbol{K},<^{*}\right\rangle$ be the Fraïssé limit of $\mathcal{K}^{*}$. Clearly $<^{*} \in \operatorname{LO}(K)$. We shall show that the uniform measure $\mu$ is supported by the generic orbit, which is the $\Gamma$-orbit of $<^{*}$ in the $\Gamma$-space $\mathrm{LO}(K)$.

As we have seen in the proof of the preceding proposition, the $\Gamma$-orbit of $<{ }^{*}$ consists of all $<$ in $\mathrm{LO}(K)$ that satisfy the following property:

Given $\boldsymbol{A}^{*} \subseteq \boldsymbol{B}^{*}$ in $\mathcal{K}^{*}$ and an embedding $\pi: \boldsymbol{A}^{*} \rightarrow\langle\boldsymbol{K},<\rangle$, there is an embedding $\rho: \boldsymbol{B}^{*} \rightarrow\langle\boldsymbol{K},<\rangle$ extending $\pi$.

Since $\mathcal{K}^{*}$ is countable (up to isomorphism), it is enough to show that for each given $\boldsymbol{A}^{*} \subseteq \boldsymbol{B}^{*}$ in the class $\mathcal{K}^{*}$, where $\boldsymbol{A}^{*}=\left\langle\boldsymbol{A},<_{\boldsymbol{A}}\right\rangle$ and $\boldsymbol{B}^{*}=\left\langle\boldsymbol{B},<_{\boldsymbol{B}}\right\rangle$, and any embedding $\pi_{\boldsymbol{A}}: \boldsymbol{A} \rightarrow \boldsymbol{K}$, if we let $\left(\pi_{\boldsymbol{A}}\right)_{*}(\boldsymbol{A})=: \boldsymbol{A}_{0} \subseteq \boldsymbol{K}$ and also $\left(\pi_{\boldsymbol{A}}\right)_{*}\left(<_{\boldsymbol{A}}\right)=:<_{0}$, then the set of all $<\in \mathrm{LO}(K)$ with $<_{0} \subseteq<$ for which there is no embedding $\pi_{\boldsymbol{B}}: \boldsymbol{B} \rightarrow \boldsymbol{K}$ extending $\pi_{\boldsymbol{A}}$ with $\left(\pi_{\boldsymbol{B}}\right)_{*}\left(<_{\boldsymbol{B}}\right) \subseteq<$ is $\mu$-null.

Since $\mathcal{K}$ satisfies the strong amalgamation property (see [KPT, Section 2 and 5.3] for the definition and this result), for each $n \geq 1$, we can find $\boldsymbol{B}_{1}, \ldots, \boldsymbol{B}_{n} \subseteq \boldsymbol{K}$ such that $\boldsymbol{A}_{0} \subseteq \boldsymbol{B}_{i}$ for $1 \leq i \leq n$ and $B_{i} \cap B_{j}=A_{0}$ if $i \neq j$, and isomorphisms $\pi_{i}: \boldsymbol{B} \rightarrow \boldsymbol{B}_{i}$ extending $\pi_{\boldsymbol{A}}$. Let $<_{i}$ be the image of $<_{\boldsymbol{B}}$ by $\pi_{i}$. Thus $<_{0} \subseteq<_{i}$ for $1 \leq i \leq n$. It is thus enough to show that the $\mu$-measure of the set of $<\in \operatorname{LO}(K)$ with $<\supseteq<_{0}$ but $<\nsupseteq<_{i}, \forall 1 \leq i \leq n$, tends to 0 as $n \rightarrow \infty$.

When the language of $\mathcal{K}$ is relational, then it is clear that it is enough to restrict ourselves to pairs $\boldsymbol{A}^{*}, \boldsymbol{B}^{*}$ as above where $|B|=|A|+1$. In this case, we can complete the proof as follows:

Let $A:=\left\{a_{1}, \ldots, a_{k}\right\}$ and $B=\left\{a_{1}, \ldots, a_{k}, b\right\}$, where $a_{1}<_{\boldsymbol{A}} a_{2}<_{\boldsymbol{A}} a_{3}<_{\boldsymbol{A}} \cdots<_{\boldsymbol{A}} a_{k}$. Then one of the following holds:

$$
b<_{\boldsymbol{B}} a_{1}, a_{1}<_{\boldsymbol{B}} b<_{\boldsymbol{B}} a_{2}, a_{2}<_{\boldsymbol{B}} b<_{\boldsymbol{B}} a_{3}, \ldots, a_{k-1}<_{\boldsymbol{B}} b<_{\boldsymbol{B}} a_{k} \text {, or } a_{k}<b .
$$

Assume $a_{1}<_{\boldsymbol{B}} b<_{\boldsymbol{B}} a_{2}$, the argument being similar in all the other cases. Let $b_{i}:=\pi_{i}(b)$ for $1 \leq i \leq n$ and $\pi_{\boldsymbol{A}}\left(a_{j}\right)=\bar{a}_{j}$ for $1 \leq j \leq k$. Then

$$
\begin{aligned}
& \left\{<\in \operatorname{LO}(K) ;<_{0} \subseteq<\& \forall 1 \leq i \leq n\left(<_{i} \not \subset\right)\right\} \\
\subseteq & \left\{<\in \operatorname{LO}(K) ;<_{0} \subseteq<\& \forall 1 \leq i \leq n\left(b_{i}<\bar{a}_{1} \vee \bar{a}_{2}<b_{i}\right)\right\} \\
\subseteq & \left.\bigcup N_{\boldsymbol{C},<} ;<\in \operatorname{LO}(C) \& \bar{a}_{1}<\bar{a}_{2} \& \forall 1 \leq i \leq n\left(b_{i}<\bar{a}_{1} \vee \bar{a}_{2}<b_{i}\right)\right\},
\end{aligned}
$$

where $\boldsymbol{C}$ is the substructure of $\boldsymbol{K}$ with universe $C=\left\{\bar{a}_{1}, \bar{a}_{2}, b_{1}, \ldots, b_{n}\right\}$. Since $\mu\left(N_{\boldsymbol{C},<}\right)=$ $\frac{1}{(n+2) !}$ for each $<\in \operatorname{LO}(C)$, it is enough to show that

$$
\frac{\mid\left\{<\in \operatorname{LO}(C) ; \bar{a}_{1}<\bar{a}_{2} \& \forall 1 \leq i \leq n\left(b_{i}<\bar{a}_{1} \vee \bar{a}_{2}<b_{i}\right\} \mid\right.}{(n+2) !} \rightarrow 0
$$


as $n \rightarrow \infty$. But simple counting shows that numerator is equal to $(n+1)$ !, so this ratio is equal to $\frac{1}{n+2} \rightarrow 0$, and the proof is complete.

In the general case, we have $A=\left\{a_{1}, \ldots, a_{k}\right\}$ and $B=\left\{a_{1}, \ldots, a_{k}, b_{1}, \ldots, b_{m}\right\}$. Then if $b_{l}^{i}:=\pi_{i}\left(b_{l}\right)(1 \leq i \leq n, 1 \leq l \leq m)$ and $\pi_{\boldsymbol{A}}\left(a_{j}\right)=: \bar{a}_{j}$, let $C:=\left\{\bar{a}_{1}, \ldots, \bar{a}_{k}\right\} \cup \bigcup_{i=1}^{n}\left\{b_{1}^{i}, \ldots, b_{m}^{i}\right\}$. Then the $\mu$-measure of

$$
\left\{<\in \mathrm{LO}(K) ;<_{0} \subseteq<\text { and } \forall 1 \leq i \leq n\left(<_{i} \not \subset\right)\right\}
$$

is equal to

$$
\frac{\mid\left\{<\in \operatorname{LO}(C) ;<_{0} \subseteq<\text { and } \forall 1 \leq i \leq n\left(<_{i} \nsubseteq<\right)\right\} \mid}{|\operatorname{LO}(C)|},
$$

so it is enough to show that this ratio goes to 0 as $n$ goes to infinity. This is a consequence of the following lemma.

Lemma 14.5 Let $X=\left\{x_{1}, \ldots, x_{k}\right\}$ and $Y=\left\{y_{1}, \ldots, y_{m}\right\}$ be two disjoint sets and let $<_{*}$ be an ordering of $X \sqcup Y$. Consider the set $X+n Y:=X \sqcup \bigsqcup_{i=1}^{n} Y^{i}$, where $Y^{i}:=\left\{y_{1}^{i}, \ldots, y_{m}^{i}\right\}$, consisting of the union of $X$ and $n$ disjoint copies of $Y$. Let $<_{i}$ be the copy of $<_{*}$ on $X \sqcup Y^{i}$. Then, for the uniform probability measure on $\mathrm{LO}(X+n Y)$, the probability that a linear ordering $<$ on $X+n Y$ extends $<_{*}\left\lceil X\right.$ but $<\nsupseteq<_{i}$ for every $1 \leq i \leq n$, tends to 0 as $n \rightarrow \infty$.

Proof. This was proved first by Padraic Bartlett with another method. One way to generate a uniform ordering on $X+n Y$ is to assign each element $t \in X+n Y$ an independent random variable $U_{t}$, uniform in $[0,1]$. The order $<$ is then induced from $[0,1]$.

The event that $<$ extends $<_{*} \mid X$ depends only on $\left\langle U_{t} ; t \in X\right\rangle$, and is equivalent to those being in the right order. Conditioned on $\left\langle U_{t} ; t \in X\right\rangle$, the restrictions of $<$ to $X \sqcup Y^{i}$ are independent, and each has non-zero probability $q=q\left(\left\langle U_{t} ; t \in X\right\rangle\right)$ of being $<_{i}$. Thus the conditional probability that $<$ extends $<_{*}\left\lceil X\right.$ but not any $<_{i}$ is $(1-q)^{n}$.

Since $q$ is almost surely non-zero, after taking expectation with respect to $\left\langle U_{t} ; t \in X\right\rangle$, this tends to 0 as $n \rightarrow \infty$ by the bounded convergence theorem.

Many of the examples of uniquely ergodic automorphism groups that we discussed earlier satisfy the conditions of the preceding theorem, so the unique invariant measure in each minimal flow concentrates on the generic orbit. These include the automorphism groups mentioned in Theorem 11.2.

\section{Some Open Problems}

The preceding work suggests a number of open problems.

Question 15.1 (Unique Ergodicity Problem) Let $\Gamma$ be an amenable Polish group with metrizable universal minimal flow. Is $\Gamma$ uniquely ergodic? 
Recall that the universal minimal flow of a countable group is not metrizable, so infinite countable groups, which, as we mentioned in the introduction, are not uniquely ergodic, do not provide counterexamples (and probably the same holds for non-compact, locally compact groups).

One can even consider a more general version of Question 15.1 for Polish groups that need not be amenable: If $\Gamma$ is a Polish group with metrizable universal minimal flow, then does every minimal flow of $\Gamma$ have at most one invariant measure?

Question 15.2 (Generic Point Problem) Let $\Gamma$ be a Polish group with metrizable universal minimal flow. Does $\Gamma$ have the generic point property?

Finally we have the following stronger version of the first problem.

Question 15.3 (Unique Ergodicity-Generic Point Problem) Let $\Gamma$ be an amenable Polish group with metrizable universal minimal flow. Is it true that $\Gamma$ is uniquely ergodic and has the generic point property and moreover for every minimal $\Gamma$-flow, the unique invariant measure is supported by the generic orbit?

Recent work of Nguyen Van Thé and Tsankov may be relevant to these problems for the case when $\Gamma$ is the automorphism group of a Fraïssé structure $\boldsymbol{K}$.

As we mentioned earlier in Section 9, there are examples of Fraïssé classes $\mathcal{K}$ that have no companions at all. However, Nguyen Van Thé [N2] developed a more general notion of expansion $\mathcal{K}^{*}$ for a given Fraïssé class $\mathcal{K}$ in a language $L$. Such an expansion is obtained by taking $L^{*}$ to be a language obtained from $L$ by adding not merely a single binary relation symbol $<$, but instead a finite or infinite (countable) family of relation symbols (of various arities) $\left(R_{i}\right)_{i \in I}$. It is shown in [N2] that the basic theory of [KPT] goes through in this more general context, provided the class $\mathcal{K}^{*}$ is precompact, i.e., every structure in $\mathcal{K}$ has only finitely many expansions in $\mathcal{K}^{*}$ (this will be automatically true if one adds only finitely many symbols to $L$ to form $L^{*}$ ). In particular, if $\mathcal{K}$ admits a precompact companion $\mathcal{K}^{*}$ (i.e., such an expansion that satisfies the Ramsey Property and the analog of the Ordering Property in this context, called the Expansion Property), then an analogous metrizable space $X_{\mathcal{K}^{*}}$ is the universal minimal flow of the automorphism group of the Fraïssé limit of $\mathcal{K}$.

Nguyen Van Thé and Tsankov (private communication, 2012) have now shown that for a Fraïssé class $\mathcal{K}$ with Fraïssé limit $\boldsymbol{K}$ and $\Gamma:=\operatorname{Aut}(\boldsymbol{K})$, the following are equivalent:

- $\Gamma$ has metrizable universal minimal flow with a comeager orbit,

- $\mathcal{K}$ admits a precompact expansion with the Ramsey Property and the Expansion Property.

Such precompact companions have been computed for: (i) the class of local orders, (ii) the age of the Fraïssé directed graph $S(3)$ (see [N2]) and (iii) the class of boron tree structures (see $[J]$ ). (In all these cases, the language $L^{*}$ turns out to be finite.) For (i) it was shown 
in [KS] that the corresponding automorphism group is not amenable and the same has been proved for (ii) and (iii) by Andrew Zucker.

Finally, let again $\left(\mathcal{K}, \mathcal{K}^{*}\right)$ be an excellent pair, with $\boldsymbol{K}:=\operatorname{Flim}(\mathcal{K})$ and $\Gamma:=\operatorname{Aut}(\boldsymbol{K})$ amenable. In all the cases that we have been able to prove unique ergodicity for $\Gamma$, it turned out that the unique $\Gamma$-invariant measure on $X_{\mathcal{K}^{*}}$ was the uniform measure given by $\mu\left(N_{\boldsymbol{A},<}\right):=\frac{1}{k(\boldsymbol{A})}$, where $k(\boldsymbol{A}):=\left|\left\{<;\langle\boldsymbol{A},<\rangle \in \mathcal{K}^{*}\right\}\right|$ is the cardinality of the set of $\mathcal{K}^{*}$-admissible orderings on $\boldsymbol{A}$. One can ask whether this is a general phenomenon.

Question 15.4 Let $\left(\mathcal{K}, \mathcal{K}^{*}\right)$ be an excellent pair with $\boldsymbol{K}:=\operatorname{Flim}(\mathcal{K})$ and let $\Gamma:=\operatorname{Aut}(\boldsymbol{K})$ be amenable. Is there a (necessarily unique) $\Gamma$-invariant measure $\mu$ on $X_{\mathcal{K}^{*}}$ satisfying $\mu\left(N_{\boldsymbol{A},<}\right)=\frac{1}{k(\boldsymbol{A})}$ ?

Notice that this is equivalent to asking the following: Let $\left(\mathcal{K}, \mathcal{K}^{*}\right)$ be as in the previous problem. Is it true that for any $\boldsymbol{A} \subseteq \boldsymbol{B}$ in $\mathcal{K}$, every $\mathcal{K}^{*}$-admissible ordering on $\boldsymbol{A}$ has the same number of extensions to a $\mathcal{K}^{*}$-admissible ordering on $\boldsymbol{B}$ ?

\section{References}

[BK] H. Becker and A.S. Kechris (1996). The Descriptive Set Theory of Polish Group Actions, Cambridge Univ. Press.

[BHV] B. Bekka, P. de la Harpe and A. Valette (2008). Kazhdan's Property (T), Cambridge Univ. Press.

[Fr] R. Fraïssé (1954). Sur l'extension aux relations de quelques propriétés des ordres, Ann. Sci. Ecole Norm. Sup. (3) 71, 363-388.

[Gl] E. Glasner (2003). Ergodic Theory via Joinings, Amer. Math. Soc.

[GW1] E. Glasner and B. Weiss (2002). Minimal actions of the group $S(\mathbb{Z})$ of permutations of the integers, Geom. Funct. Anal. 12, 964-988.

[GW2] E. Glasner and B. Weiss (2003). The universal minimal system for the group of homeomorphisms of the Cantor set, Fund. Math. 176, 277-289.

[He1] B. Herwig (1995). Extending partial isomorphisms on finite structures, Combinatorica 15 (3), 365-371.

[He2] B. Herwig (1998). Extending partial isomorphisms for the small index property of many w-categorical structures, Israel J. Math. 107, 93-123.

[Ho] W. Hodges (1993). Model Theory, Cambridge Univ. Press.

[Hr] E. Hrushovski (1992). Extending partial isomorphisms of graphs, Combinatorica 12, 411-416. 
[J] J. Jasiński (2011). Hrushovski and Ramsey Properties of Classes of Finite Inner Product Structures, Finite Euclidean Metric Spaces, and Boron Trees, Ph.D. Thesis, Univ. of Toronto.

[K] A.S. Kechris (1995). Classical Descriptive Set Theory, Springer.

[KPT] A.S. Kechris, V.G. Pestov, and S. Todorcevic (2005). Fraïssé limits, Ramsey theory, and topological dynamics of automorphism groups, Geom. Funct. Anal. 15, 106189 .

[KR] A.S. Kechris and C. Rosendal (2007). Turbulence, amalgamation, and generic automorphisms of homogeneous structures, Proc. London Math. Soc. 94 (3), 302-350.

[KS] A.S. Kechris and M. Sokić (2011). Dynamical properties of the automorphism groups of the random poset and random distributive lattice, Fund. Math., to appear.

[McD] C. McDiarmid (1989). On the method of bounded differences. In Siemons, J., editor, Surveys in Combinatorics, 1989, volume 141 of London Math. Soc. Lecture Note Ser., pages 148-188. Cambridge Univ. Press, Cambridge. Papers from the Twelfth British Combinatorial Conference held at the University of East Anglia, Norwich, 1989.

[Mec] M.W. Meckes (2010). Positive definite metric spaces. Preprint, http://www. arxiv . org/abs/1012.5863.

[MT] J. Melleray and T. Tsankov (2011). Generic representations of abelian groups and extreme amenability. Preprint, http://www. arxiv.org/abs/1107.1698v1.

[Mo] J.T. Moore (2011). Amenability and Ramsey theory. Preprint, http://www . arxiv . org/abs/1106.3127v4.

[NR] J. Nešetřil and V. Rödl (1978). On a probabilistic graph-theoretical method. Proc. Amer. Math. Soc. 72, 417-421.

[N1] L. Nguyen Van Thé (2010). Structural Ramsey theory of metric spaces and topological dynamics of isometry groups, Memoirs of the Amer. Math. Soc. 206, No. 968.

[N2] L. Nguyen Van Thé (2012). More on the Kechris-Pestov-Todorcevic correspondence: Precompact expansions. Preprint, http://www .arxiv.org/abs/1201.12708v1.

[Pe1] V. Pestov (1998). On free actions, minimal flows and a problem by Ellis, Trans. Amer. Math. Soc. 350 (10), 4149-4165.

[Pe2] V. Pestov (2002). Ramsey-Milman phenomenon, Urysohn metric spaces, and extremely amenable groups, Israel J. Math. 127, 317-357. 
[Sch] I.J. Schoenberg (1938). Metric spaces and positive definite functions. Trans. Amer. Math. Soc. 44, 522-536.

[So] S. Solecki (2005). Extending partial isometries, Israel J. Math. 150, 315-332.

[W] B. Weiss (2012). Minimal models for free actions, Contemp. Math. 567, 249-264.

\author{
Omer Angel \\ Department of Mathematics \\ University of British Columbia \\ Vancouver, British Columbia V6T 1 Z2 \\ angel@math . ubc . ca \\ Alexander S. Kechris \\ Department of Mathematics \\ California Institute of Technology \\ Pasadena, CA 91125 \\ kechris@caltech.edu \\ Russell Lyons \\ Department of Mathematics \\ 831 E 3rd St \\ Indiana University \\ Bloomington, IN 47405-7106 \\ rdlyons@indiana.edu \\ mypage.iu.edu/ rdlyons
}

